\title{
KIC 5950759: a high-amplitude $\delta$ Sct star with amplitude and frequency modulation near the terminal age main sequence ${ }^{\star}$
}

\author{
D. M. Bowman, ${ }^{1} \dagger$ J. Hermans,${ }^{2,1}$ J. Daszyńska-Daszkiewicz, ${ }^{3}$ D. L. Holdsworth, ${ }^{4}$ \\ A. Tkachenko, ${ }^{1}$ S. J. Murphy, ${ }^{5,6}$ B. Smalley, ${ }^{7}$ D. W. Kurtz ${ }^{8,4}$ \\ ${ }^{1}$ Institute of Astronomy, KU Leuven, Celestijnenlaan 200D, 3001 Leuven, Belgium \\ 2 Centre for mathematical Plasma Astrophysics, KU Leuven, 3001, Leuven, Belgium \\ ${ }^{3}$ Astronomical Institute, University of Wrocław, ul. Kopernika 11, 51-622 Wrocław, Poland \\ ${ }^{4}$ Jeremiah Horrocks Institute, University of Central Lancashire, Preston PR1 2HE, UK \\ ${ }^{5}$ Sydney Institute for Astronomy (SIfA), School of Physics, University of Sydney, NSW 2006, Australia \\ 6 Stellar Astrophysics Centre, Department of Physics and Astronomy, Aarhus University, DK-8000 Aarhus C, Denmark \\ ${ }^{7}$ Astrophysics Group, Lennard-Jones Laboratories, Keele University, Staffordshire ST5 5BG, UK \\ 8 Centre for Space Research, Physics Department, North-West University, Mahikeng 2745, South Africa
}

Accepted 2021 April 16. Received 2021 April 13; in original form 2021 March 16

\begin{abstract}
Amongst the intermediate mass pulsating stars known as $\delta$ Sct stars is a subset of highamplitude and predominantly radial-mode pulsators known as high-amplitude $\delta$ Sct (HADS) stars. From more than $2000 \delta$ Sct stars observed by the Kepler space mission, only two HADS stars were detected. We investigate the more perplexing of these two HADS stars, KIC 5950759. We study its variability using ground- and space-based photometry, determine its atmospheric parameters from spectroscopy and perform asteroseismic modelling to constrain its mass and evolutionary stage. From spectroscopy, we find that KIC 5950759 is a metal-poor star, which is in agreement with the inferred metallicity needed to reproduce its pulsation mode frequencies from non-adiabatic pulsation models. Furthermore, we combine ground-based WASP and Kepler space photometry, and measure a linear change in period of order $\dot{P} / P \simeq 10^{-6} \mathrm{yr}^{-1}$ for both the fundamental and first overtone radial modes across a time base of several years, which is at least two orders of magnitude larger than predicted by evolution models, and is the largest measured period change in a $\delta$ Sct star to date. Our analysis indicates that KIC 5950759 is a metal-poor HADS star near the short-lived contraction phase and the terminal-age main sequence, with its sub-solar metallicity making it a candidate SX Phe star. KIC 5950759 is a unique object amongst the thousands of known $\delta$ Sct stars and warrants further study to ascertain why its pulsation modes are evolving remarkably faster than predicted by stellar evolution.
\end{abstract}

Key words: asteroseismology - stars: oscillations - stars: variables: $\delta$ Scuti - stars: variables: HADS - stars: variables: SX Phe - stars: individual: KIC 5950759

\section{INTRODUCTION}

One of the goals of astrophysics is to improve models of stellar evolution by using constraints from observations. Understanding the physical processes inside a star, such as convection, rotation, angular momentum transport and mixing are of vital importance in improving stellar evolutionary models (Maeder \& Meynet 2000; Maeder 2009). This is especially true for intermediate- and highmass stars since they are born with a convective core, and the the-

* Based on observations made with the WHT operated on the island of La Palma by the Isaac Newton Group in the Spanish Observatorio del Roque de los Muchachos of the Instituto de Astrofísica de Canarias.

† E-mail: dominic.bowman@kuleuven.be oretical uncertainties associated with convection and mixing in the core-envelope boundary region strongly influence their post-main sequence evolution (Kippenhahn et al. 2012). Similarly, when such stars are close to depleting the hydrogen fuel in their cores, they enter an overall contraction phase to restore hydrostatic equilibrium, which results in a blue hook in the Hertzsprung-Russell (HR) diagram (Iben 1967). The location and morphology of this blue hook depends on the mass, metallicity, rotation rate and the properties of convection within the star. One of the most promising techniques to mitigate these uncertainties and ultimately improve the predictive power of stellar evolution models is using observations of stellar pulsations. In this technique, known as asteroseismology, the resonant pulsation frequencies of a star are used to constrain its interior 
properties from a quantitative comparison to stellar models containing different input physics (Aerts et al. 2010; Aerts 2021).

Amongst intermediate mass Population I stars of spectral type $\mathrm{A}$ and $\mathrm{F}$ is a group of pulsating stars known as $\delta$ Sct stars, which have pulsation periods that range from $\sim 15$ min to several hours (Breger 2000b; Aerts et al. 2010; Bowman \& Kurtz 2018). They are located at the intersection of the main sequence and the classical instability strip in the HR diagram and have temperatures that range from approximately 7500 to $9500 \mathrm{~K}$ at the zero-age main sequence (ZAMS) and from approximately 6500 to $8500 \mathrm{~K}$ at the terminalage main-sequence (TAMS; Bowman \& Kurtz 2018; Murphy et al. 2019). Thousands of $\delta$ Sct stars are known based on ground-based observations and surveys (Rodríguez et al. 2000; Jayasinghe et al. 2020) and more recently space-based telescopes including Kepler (Borucki et al. 2010; Uytterhoeven et al. 2011; Balona \& Dziembowski 2011) and TESS (Ricker et al. 2015; Antoci et al. 2019). The parameter space covered by $\delta$ Sct stars in the HR diagram is of great importance for testing stellar evolution models. They cover the transition region from slowly-rotating low-mass stars with radiative cores and thick convective envelopes $\left(M \leq 1.5 \mathrm{M}_{\odot}\right)$ to rapidly-rotating intermediate mass stars with convective cores and predominantly radiative envelopes $\left(M \geq 2.5 \mathrm{M}_{\odot}\right)$. This transition in stellar structure allows many different aspects of physics to be investigated, including pulsation, rotation, magnetic fields and chemical peculiarities (see Murphy 2014 for a review).

Pulsations in $\delta$ Sct stars are typically excited by the opacitydriven heat engine $\kappa$ mechanism operating in the He II ionization zone, which produces radial $(\ell=0)$ and non-radial $(\ell>0)$ pressure (p) modes with pulsation periods between $\sim 15$ min and several hours (Breger 2000b; Pamyatnykh 2000). Many $\delta$ Sct stars are also observed to exhibit low-frequency variability caused by gravity (g) modes (see e.g. Balona \& Dziembowski 2011), which are not predicted to be excited in early-A main-sequence stars without a significant modification to stellar opacity tables (Dupret et al. 2005; Balona et al. 2015). Recent work on the excitation of pulsations in these stars has revealed that turbulent pressure is also important for exciting higher radial order p modes (Antoci et al. 2014), and that the efficiency of mixing and the helium abundance in the nearsurface layers of $\delta$ Sct stars plays an important role (Antoci et al. 2019; Murphy et al. 2020).

Within the pulsator group of the $\delta$ Sct stars, a small subset is observed to exhibit large pulsation amplitudes; these are known as high-amplitude $\delta$ Sct (HADS) stars (McNamara 1997, 2000b) ${ }^{1}$. The HADS stars are in general slow rotators with projected surface rotational velocities of $v \sin i \leq 40 \mathrm{~km} \mathrm{~s}^{-1}$, which is in contrast to the moderate and high rotation rates of main-sequence A stars (Zorec \& Royer 2012; Niemczura et al. 2015). In terms of their pulsation properties, HADS stars predominantly pulsate in the fundamental and/or first overtone radial p mode (Petersen \& ChristensenDalsgaard 1996; Breger 2000a; McNamara 2000b; Rodríguez et al. 2000; Derekas et al. 2009). The HADS stars were investigated by McNamara (2000b), who classified them as $\delta$ Sct stars with large peak-to-peak light amplitudes (typically exceeding $0.3 \mathrm{mag}$ ) and pulsation periods between 1 and $6 \mathrm{hr}$. However, this historical classification is based only on inspection of the light curve, so it remains unclear why these stars are so rare and how they are distinct from their low amplitude $\delta$ Sct star cousins. For example, Lee et al. (2008)

\footnotetext{
1 McNamara (1997) credit the classification of HADS stars as a distinct group of pulsators to an unpublished Harvard university dissertation of $\mathrm{H}$. Smith in 1955.
}

estimated that less than 1 per cent of pulsating A and F stars are HADS stars. Moreover, only two HADS stars were found within the sample of more than $10000 \mathrm{~A}$ and F stars observed by the Kepler mission (Bowman et al. 2016; Balona 2016). Some HADS stars have been shown to be members of multiple systems based on their measured radial velocities (e.g. Derekas et al. 2009), although a complete census of binarity amongst HADS stars is lacking owing to their rarity.

In the literature HADS stars have been sometimes thought of as transition objects between the main sequence $\delta$ Sct stars and the more-evolved classical (i.e. radial) pulsators, as they exhibit a tight period-luminosity relationship similar to Cepheid variables (see e.g. Soszyński et al. 2008; Poleski et al. 2010). On the other hand, a period-luminosity relationship for $\delta$ Sct stars investigated by Ziaali et al. (2019) contained considerably more scatter owing to these stars typically having both radial and non-radial modes with comparable amplitudes that exhibit long-term amplitude modulation (Bowman et al. 2016). Because of their rarity, there have only been a few detailed studies of HADS stars in the literature, which aimed to determine if these stars are distinct from $\delta$ Sct stars. For example, Petersen \& Christensen-Dalsgaard (1996) claimed that HADS stars are able to pulsate at significantly higher amplitudes because they are in a post-main sequence stage of stellar evolution. On the other hand, Breger (2000a) conjectured that the slow rotation in these stars facilitates the high amplitude pulsations, which are not able to be excited in rapidly rotating $\delta$ Sct stars because of the deformation from spherical symmetry.

The Population II counterparts of $\delta$ Sct stars are known as SX Phe stars, named after the first such star to show highamplitude radial pulsations (Frolov \& Irkaev 1984), although such high-amplitude pulsation has been known since Goodricke (1783). SX Phe stars are also located within the classical instability strip in the HR diagram, have similar pulsation frequencies to $\delta$ Sct stars, and dominant radial pulsation modes with relatively large amplitudes like HADS stars (Balona \& Nemec 2012). However, they sometimes have sub-solar metallicities and large spatial motions (McNamara 2000b; Nemec et al. 2017). Interestingly, SX Phe stars are known to exhibit period (i.e. pulsation frequency) changes when monitored over several years (Coates et al. 1982; Thompson \& Coates 1991; Rodríguez et al. 2007; Yang et al. 2012; Murphy et al. 2013b), and yet these changes are sometimes observed as sudden jumps in period rather than smooth variation over time (e.g. Rodríguez et al. 1995). Therefore, it remains unclear if the period changes and associated amplitude modulation of pulsation modes are directly caused by stellar evolution. Recently, DaszyńskaDaszkiewicz et al. (2020) performed detailed seismic modelling of the prototype of this class, SX Phe, constrained its fundamental parameters and confirmed its post-main sequence evolutionary phase thanks to high-precision photometry assembled by the TESS mission.

In this paper, we present the study of the pulsating star KIC 5950759, which is one of only two HADS stars observed by the Kepler space telescope during its nominal 4-yr mission. In section 2 we use Kepler and WASP photometry to extract its pulsation mode frequencies and measure their long-term variability. In section 3, we present our spectroscopic analysis of KIC 5950759, and in section 4 we use Gaia parallaxes to calculate its luminosity. We perform forward asteroseismic modelling of KIC 5950759 in section 5 , and we conclude in section 6 . 
Table 1. Stellar parameters of KIC 5950759 from photometric catalogues and spectroscopy from this work (see text for details), including the effective temperature $\left(T_{\text {eff }}\right)$, surface gravity (log $\left.g\right)$ and metallicity $([\mathrm{M} / \mathrm{H}])$.

\begin{tabular}{cccl}
\hline $\begin{array}{c}\mathrm{T}_{\text {eff }} \\
(\mathrm{K})\end{array}$ & $\log g$ & $\begin{array}{c}{[\mathrm{M} / \mathrm{H}]} \\
(\mathrm{dex})\end{array}$ & \multicolumn{1}{c}{ Reference } \\
\hline $7840 \pm 300$ & $4.03 \pm 0.25$ & $-0.07 \pm 0.25$ & Brown et al. (2011) \\
$8070 \pm 280$ & $4.03 \pm 0.40$ & $-0.07 \pm 0.30$ & Huber et al. (2014) \\
\hline $7340 \pm 220$ & $2.9 \pm 1.3$ & $-1.18 \pm 0.30$ & this work \\
$7470 \pm 255$ & $3.6 \pm 1.2$ & $-0.65 \pm 0.45$ & this work \\
\hline
\end{tabular}

\section{PHOTOMETRY}

KIC 5950759 was first identified as a variable star with a dominant period of $0.0703176 \mathrm{~d}$ by Pigulski et al. (2009) using ASAS data. Later, it was identified as one of only two HADS stars in the nominal field of view of the Kepler mission (Bowman et al. 2016; Balona 2016), which satisfied the criterion of having large peak-to-peak light amplitude variations typical of such stars (McNamara 2000b). The parameters of KIC 5950759 from the Kepler Input Catalogue (KIC; Brown et al. 2011) and the revised values from Huber et al. (2014), which are similar to the updated values of Mathur et al. (2017), are provided in Table 1.

\subsection{Kepler Photometry}

In this work, light curves from the multi-scale Maximum A Priori Pre-Search Data Conditioning (msMAP PDC) pipeline developed by the Kepler Science Office (Stumpe et al. 2012; Smith et al. 2012) are used, which are publicly available from the Mikulski Archive for Space Telescopes (MAST) ${ }^{2}$. KIC 5950759 was observed between Q1 and Q17 of the nominal Kepler mission in its long cadence (LC; $29.5 \mathrm{~min}$ ) mode, for a total of $1460 \mathrm{~d}$. It was also observed in the first month of Q4 in short cadence (SC; 59 s) mode for a total of $31.1 \mathrm{~d}$. The effective Nyquist frequencies of the LC and SC data are 24.47 and $727.35 \mathrm{~d}^{-1}$, respectively. The Kepler light curves were converted into stellar magnitudes and long-period instrumental systematics were removed by means of subtracting a low-order polynomial from each LC data quarter.

From the LC and SC light curves amplitude spectra were calculated by means of a discrete Fourier transform (Kurtz 1985). The amplitude spectrum of the single month of SC Kepler data is shown in the top panel of Fig. 1, in which the two high-amplitude pulsation modes, $\nu_{1}$ and $\nu_{2}$, and their dominant combination frequencies are labelled. The two high-amplitude modes have a period (and frequency) ratio of 0.77554 identifying them as the fundamental and first overtone radial modes (Breger 2000b). In $\delta$ Sct stars with such high-amplitude (radial) pulsation modes, it is typical to observe many high-order harmonics and combination frequencies i.e. frequencies that can be identified using $n \nu_{1} \pm m \nu_{2}$, in which $n$ and $m$ are integers (Pápics 2012; Kurtz et al. 2015; Balona 2016). The physical causes of combination frequencies include the stellar medium not responding linearly to a pulsation wave, or that the flux variability caused by waves is not a linear transformation of the temperature variability (see e.g. Brickhill 1992). These phenomena are generally grouped together into what is called a non-linear distortion model (e.g. Degroote et al. 2009; Bowman 2016). On the other hand, some combination frequencies represent resonantly

2 http://archive.stsci.edu/kepler/

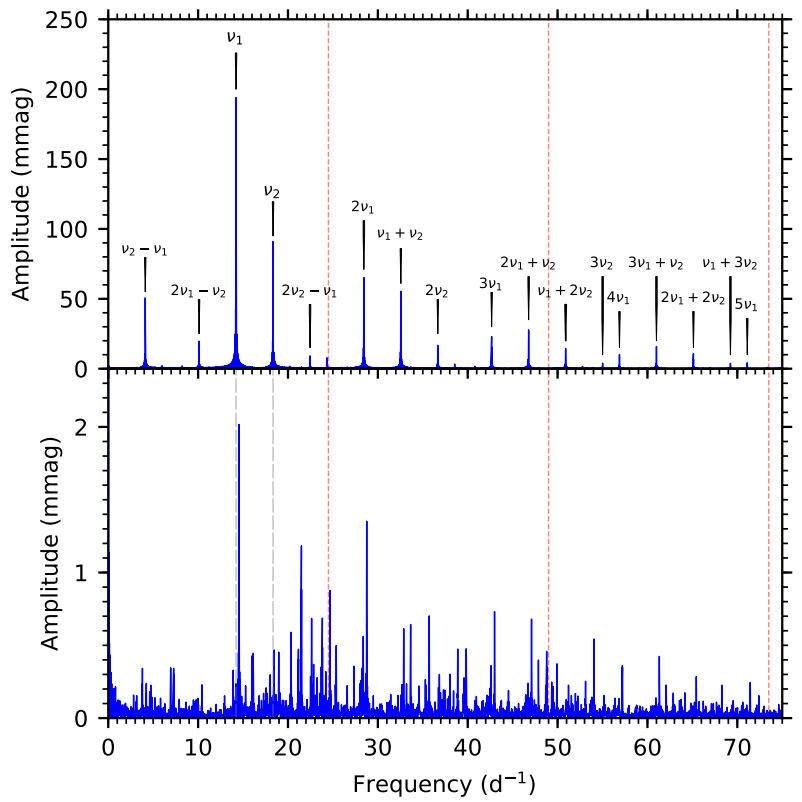

Figure 1. Top panel: the amplitude spectrum calculated from the one month of SC Kepler data for KIC 5950759, in which the two dominant pulsation modes, $\nu_{1}$ and $\nu_{2}$, and their high-amplitude combination frequencies are labelled. Bottom panel: the residual amplitude spectrum of the same SC Kepler data after $\nu_{1}$ and $\nu_{2}$ and all their significant combination frequencies have been removed by pre-whitening. The long-dashed grey lines denote the location of $\nu_{1}$ and $\nu_{2}$ in the bottom panel, and the short-dashed red lines indicate integer multiples of the Kepler LC Nyquist frequency to demonstrate that considerable variance remains beyond the Kepler LC Nyquist frequency.

Table 2. Frequency, amplitude, and phase of the two dominant pulsation modes, $\nu_{1}$ and $\nu_{2}$, in KIC 5950759, which were obtained using a non-linear least-squares fit to the 4-yr LC Kepler light curve, and the 31-d SC light curve. Pulsation phases were calculated with respect to the time zero-point $t_{0}=2455688.770$ BJD.

\begin{tabular}{cccrc}
\hline & & $\begin{array}{c}\text { Frequency } \\
\left(\mathrm{d}^{-1}\right)\end{array}$ & $\begin{array}{c}\text { Amplitude } \\
(\mathrm{mmag})\end{array}$ & \multicolumn{1}{c}{$\begin{array}{c}\text { Phase } \\
(\mathrm{rad})\end{array}$} \\
\hline \multirow{2}{*}{$\mathrm{LC}$} & $\nu_{1}$ & $14.2213938 \pm 0.0000009$ & $167.7 \pm 0.3$ & $1.685 \pm 0.002$ \\
& $\nu_{2}$ & $18.3372939 \pm 0.0000015$ & $74.1 \pm 0.3$ & $1.178 \pm 0.004$ \\
\hline \multirow{2}{*}{$\mathrm{SC}$} & $\nu_{1}$ & $14.22137 \pm 0.00005$ & $194.1 \pm 0.5$ & $1.6 \pm 0.1$ \\
& $\nu_{2}$ & $18.33725 \pm 0.00010$ & $91.2 \pm 0.5$ & $1.0 \pm 0.3$ \\
\hline
\end{tabular}

excited pulsation modes that are the result of direct mode coupling (see e.g. Breger \& Montgomery 2014; Bowman et al. 2016).

A detailed frequency analysis of both the LC and SC Kepler data of KIC 5950759 was originally performed by Bowman (2017), which focused on the two radial modes and their combination frequencies. Since the much longer 4-yr LC Kepler data set provides significantly better frequency resolution and precision, Bowman (2017) extracted and optimised the parameters of the two dominant pulsation modes, $\nu_{1}$ and $\nu_{2}$, using a multi-frequency non-linear least-squares fit to the LC Kepler light curve with the equation:

$\Delta m=\sum_{i} A_{i} \cos \left(2 \pi \nu_{i}\left(t-t_{0}\right)+\phi_{i}\right)$,

where $A_{i}$ is the amplitude, $\nu_{i}$ is the frequency, $\phi_{i}$ is the phase, $t$ is the time with respect to the zero-point $t_{0}=2455688.770$ BJD (i.e. 
the midpoint of the 4-yr Kepler light curve) for $i$ frequencies. The resultant parameters for $\nu_{1}$ and $\nu_{2}$ are provided in Table 2.

Bowman (2017) also performed a similar optimisation of the two dominant pulsation modes using Eq. 1 and a non-linear leastsquares fit to the SC Kepler data of KIC 5950759. Since the SC Kepler data has a much higher Nyquist frequency, Bowman (2017) calculated the expected frequencies of combination frequencies of $\nu_{1}$ and $\nu_{2}$ up to order of 20 (i.e. $n \nu_{1} \pm m \nu_{2}$, where $n, m \in[0,20]$ ), and optimised their amplitudes and phases using a multi-frequency linear least-squares fit, and subtracted them from the SC Kepler light curve to reveal any additional independent pulsation modes. Such high harmonic and combination orders are necessary for KIC 5950759 given the high amplitudes of the parent modes (i.e. $\nu_{1}$ and $\nu_{2}$ ) and the pristine quality of Kepler data. For example, the twentieth harmonic of $\nu_{1}$ (i.e. $20 \nu_{1}$ ) is significant in the SC data of KIC 5950759 with an amplitude of approximately $10 \mu \mathrm{mag}$. By using the approach of calculating the expected combination frequencies, any variance coinciding with the expected frequencies of combinations is removed from the light curve by means of subtracting the multi-frequency linear least-squares fit solution. The amplitude spectra of the SC Kepler light curve up to $75 \mathrm{~d}^{-1}$ both before and after removing $\nu_{1}, \nu_{2}$ and all their combination frequencies are shown in the top and bottom panels of Fig. 1, respectively, which clearly demonstrates additional variance in KIC 5950759.

Yang et al. (2018) later performed an independent frequency analysis of the available Kepler data of KIC 5950759. They confirmed the previous characterisation of $\nu_{1}$ and $\nu_{2}$ as radial modes and investigated a detected weak modulation effect present in KIC 5950759, which resulted in equally-spaced frequency multiplets around the dominant pulsation modes. The separation between the frequencies of these multiplets, $0.31923 \mathrm{~d}^{-1}$, was also detected as a significant frequency in the amplitude spectrum. Yang et al. (2018) concluded that these multiplets could be caused by amplitude modulation of the radial modes from the rotation of the $s^{3}{ }^{3}$. Such a modulation mechanism acting on radial modes has been found in some $\delta$ Sct stars (e.g. Breger et al. 2011), but its physical cause remains difficult to interpret. The inferred rotation frequency from the multiplets, $0.31925 \mathrm{~d}^{-1}$, would indicate a slow rotation rate, which is expected for HADS stars. For a discussion of this modulation effect, we refer the reader to Yang et al. (2018).

More recently, Hermans (2019) re-visited the frequency analysis of KIC 5950759 for the specific purpose of identifying additional non-radial modes. The same LC and SC light curves studied by Bowman (2017) were analysed by Hermans (2019) for consistency and comparison purposes. The LC and SC data were subjected to iterative pre-whitening, and modelled as a linear superposition of modes, as is typical for pulsating stars (see e.g. Degroote et al. 2009; Pápics et al. 2012; Van Reeth et al. 2015; Antoci et al. 2019). During each iteration, the frequency of the highest amplitude peak is determined, optimised to the light curve using Eq. (1), which yields the optimised frequency, amplitude and phase. The resulting cosinusoid model is then subtracted from the light curve and the residual time series is used as input in the next iteration. The standard stop criterion of an amplitude signal-to-noise (S/N) criterion of four (Breger et al. 1993) was used by Hermans (2019) to extract all significant frequencies, in which the noise was calculated as the

3 To be clear, this does not mean rotational splitting of radial modes, since this is not possible, but rather means that the pulsation amplitudes of the radial modes varies during the rotation phase causing equally-spaced multiplets in the amplitude spectrum. median amplitude in a window of $1 \mathrm{~d}^{-1}$ centred on the extracted frequency at each iteration.

A total of 150 significant frequencies were identified in the LC Kepler data in the frequency interval of $0 \leq \nu \leq 120 \mathrm{~d}^{-1}$, and 106 significant frequencies in the SC Kepler data of KIC 5950759 in the frequency interval $0 \leq \nu \leq 200 \mathrm{~d}^{-1}$ by Hermans (2019). Although pulsation modes with frequencies above $60 \mathrm{~d}^{-1}$ are rare in $\delta$ Sct stars (Bowman \& Kurtz 2018), the large frequency range was chosen to ensure that high frequency non-radial modes were not excluded. From these lists of extracted significant frequencies, spurious frequencies were filtered using the resolution criterion proposed by Loumos \& Deeming (1978), such that the minimal frequency separation between independent close frequencies, $\nu_{x}$ and $\nu_{y}$, satisfied

$\left|\nu_{x}-\nu_{y}\right| \geq \frac{1.5}{\Delta T}$,

where $\Delta T$ is the length of the data set. The highest amplitude peak was kept during the filtering for spurious frequencies. Combination frequencies were identified by searching for linear sum and difference frequencies, $n \nu_{i} \pm m \nu_{j}$, with the Loumos \& Deeming (1978) criterion as a tolerance and assuming that the highest-amplitude peaks within a combination family are the real pulsation mode frequencies (Kurtz et al. 2015). Although the Loumos \& Deeming (1978) criterion is not a formal frequency precision (see e.g. Lares-Martiz et al. 2020), it does act as a conservative metric for identifying combination frequencies given the added uncertainties introduced in the form of spurious frequencies from an imperfect linear least-squares pre-whitening methodology (see e.g. Degroote et al. 2009; Pápics et al. 2012; Van Reeth et al. 2015; Antoci et al. 2019). Finally, Nyquist alias frequencies were identified by crossmatching the resultant list of filtered frequencies in the LC and SC analysis, and by applying the super-Nyquist technique (Murphy et al. 2013a) to the LC Kepler data.

In total, 12 and 13 independent pulsation mode frequencies (i.e. those that cannot be explained as spurious close frequencies, combination frequencies, or Nyquist aliases) were identified from the LC and SC data in addition to $\nu_{1}$ and $\nu_{2}$ (cf. Table 2), which are given in Tables A1 and A2, respectively. Therefore, in addition to the two high-amplitude radial pulsation modes, $\nu_{1}$ and $\nu_{2}$, KIC 5950759 reveals multiple additional non-radial pulsation modes. Unfortunately, mode identification is not possible for these non-radial modes owing to their lack of regularity in the extracted frequencies. We also note that the same frequency multiplets found by Yang et al. (2018) centred on the radial mode frequencies are present in both $\mathrm{LC}$ and $\mathrm{SC}$ data, but have much lower $\mathrm{S} / \mathrm{N}$ values in the SC data. For example, the modulation frequency, $0.31925 \pm 0.00004 \mathrm{~d}^{-1}$, is only significant in the LC data because of the lower local noise level.

\subsection{WASP photometry}

Given the extremely high frequency precision provided by its highamplitude radial modes using Kepler data, Bowman (2016) identified KIC 5950759 as an excellent candidate to probe pulsation period changes. Moreover, ground-based photometric surveys prior to and during the Kepler mission have also been able to monitor its high-amplitude pulsation modes. A naturally complementary photometric survey to our analysis of the Kepler light curve of KIC 5950759 is the Wide Angle Search for Planets (WASP) project, which was a wide-field and ground-based photometric survey that aimed to find transiting exoplanets (Pollacco et al. 2006; 
Butters et al. 2010). The project had two observing sites, one at the Observatorio del Roque de los Muchachos on La Palma and the other at the Sutherland Station of the South African Astronomical Observatory (SAAO). The pixel size of each instrument is approximately 14 arcsec with observations made using broad-band filters of $400-700 \mathrm{~nm}$, which are slightly more sensitive to bluer wavelengths compared to the Kepler passband. WASP data are corrected for extinction, the colour-response of the instrument, the zero-point, and instrumental systematics using the SYSREM algorithm (Tamuz et al. 2005). The observing strategy of WASP provided two consecutive 30-s exposures at a given pointing, before moving to the next available field. Each field was typically revisited every $10 \mathrm{~min}$ on a given night, which produces a high effective Nyquist frequency (Holdsworth 2015).

The WASP survey observed KIC 5950759 for three seasons in 2007, 2009 and 2010, with light curves spanning 65.8, 127.9 and $122.9 \mathrm{~d}$, respectively. Although the photometric precision and number of data points are not as high in each WASP light curve compared to the LC Kepler data, the high-amplitude pulsation modes in KIC 5950759 are easily detectable. Moreover, during 2009 and 2010, data collection by both WASP and Kepler were concurrent. This has great advantages and allows us to use the WASP data to probe the variability in KIC 5950759 prior to the launch of the $\mathrm{Ke}$ pler space telescope, as previously demonstrated using the $\delta$ Sct star KIC 7106205 by Bowman et al. (2015). To compare simultaneous WASP and LC Kepler observations of KIC 5950759 in 2009 and 2010, the Kepler data were truncated to have the same length as the individual WASP seasons. Also, the time stamps of the WASP data, which were originally in HJD (UTC), were converted to BJD (TDB) using the PYTHON module ASTROPY (Astropy Collaboration et al. 2013,2018 ) to be fully consistent in both time scale and format with the Kepler data. An example of the concurrent LC Kepler and WASP light curves for KIC 5950759 in 2010 are shown in the top panel of Fig. 2. The lower panels in Fig. 2 show the amplitude spectra of the WASP data (right-hand panels) and the truncated Kepler data (left-hand panels).

Because of the discrete and finite sampling of an instrument's integration time, a star's pulsation modes are suppressed in amplitude because of an effect known as apodization or the amplitude suppression function (Bowman et al. 2015). The observed pulsation amplitudes can be corrected using

$$
A=A_{0} \operatorname{sinc}\left(\frac{\pi}{n}\right)=A_{0} \operatorname{sinc}\left(\frac{\pi \nu}{\nu_{\mathrm{samp}}}\right),
$$

where $A$ and $A_{0}$ are the observed and intrinsic pulsation mode amplitudes, respectively, $n$ is the number of data points per pulsation cycle, $\nu$ is the pulsation mode frequency and $\nu_{\text {samp }}$ is the instrument's sampling frequency (Murphy 2014; Bowman 2017).

A second correction arising from the difference in instrument passband is also needed, since the WASP passband is slightly more sensitive to bluer wavelengths compared to Kepler, such that observed pulsation amplitudes of $\mathrm{A}$ and $\mathrm{F}$ stars are (slightly) larger when observed at bluer wavelengths. We use the resultant value for this passband correction from Bowman et al. (2015), who estimated this correction factor to be 7.6 per cent for the late-A star KIC 7106205 - i.e. pulsation mode amplitudes are 7.6 per cent smaller in Kepler observations compared to WASP observations because of the difference in effective wavelength range of their passbands ${ }^{4}$.

4 More generally, this factor depends on the effective temperature of the
Thus we corrected the WASP and truncated Kepler data obtained in 2009 and 2010 for their respective instrument's integration time, and then transformed the WASP pulsation mode amplitudes into the Kepler passband using the passband correction factor from Bowman et al. (2015). The remaining difference between the pulsation mode amplitudes observed by WASP and Kepler in 2009 and 2010 is because of flux dilution from nearby and background stars within the larger WASP aperture (Bowman et al. 2015). A ratio of the respectively corrected WASP and Kepler mode amplitudes in 2009 and 2010 reveals an average dilution factor of $2.2 \pm 0.1-$ i.e. the integration time and passband corrected WASP amplitudes are approximately 45 per cent those of the Kepler amplitudes. Of the integration time, passband and dilution correction factors, the latter is certainly the largest effect and the largest source of uncertainty in this analysis. This is already evident in the non-corrected amplitude spectra shown in Fig. 2.

After the integration time, passband and dilution correction factors were determined and applied to the 2009 and 2010 WASP data, they were also applied to the 2007 WASP data to estimate the amplitude of the fundamental and first overtone radial modes in KIC 5950759. We provide the results for the corrected WASP amplitudes of $\nu_{1}$ and $\nu_{2}$ in the top-left and top-right panels of Fig. 3, respectively. We also divided the 4-yr Kepler data into bins of $150 \mathrm{~d}$ with 100-d overlap, following the technique employed by Bowman et al. (2016), and show the amplitude and phase as a function of fixed frequency (cf. Table 2) in Fig. 3. The quasi-sinusoidal variability in the pulsation amplitudes in the LC Kepler data has a period equal to that of the Kepler orbital period, and is caused by a changing signalto-background flux ratio caused by the rotation of the spacecraft every $90 \mathrm{~d}$. Thus, it is of instrumental and not astrophysical origin, although the amplitude is arguably constant within $2 \sigma$ regardless. Despite the relatively large amplitude uncertainties associated with the WASP data in 2007, we conclude that there is tentative evidence for a growing amplitude in the fundamental and first overtone radial modes in KIC 5950759 prior to the start of the Kepler observations.

\subsection{Period Changes}

During the main sequence and post-main sequence phases of stellar evolution, an intermediate mass star experiences an increase in radius, which increases the size of the acoustic cavity of its radial pulsation modes, hence the periods of radial modes increase with age. On the other hand, the relatively short-lived overall contraction phase near the TAMS, which results in a "blue hook" in the HR diagram for intermediate mass stars (Iben 1967; Kippenhahn et al. 2012), is a phase of evolution in which the stellar radius decreases such that the periods of radial modes are expected to decrease. Consequently, the rate of change in the periods of radial modes theoretically allow stellar evolution to be measured (Walraven et al. 1992; Breger \& Pamyatnykh 1998).

Period changes in pulsation modes have been historically studied by means of the O - C technique (Percy et al. 1980; Sterken 2005). Based on the work of Breger \& Pamyatnykh (1998), period changes in pulsation modes can be determined from

$\mathrm{O}-\mathrm{C}=\frac{1}{2}\left(\frac{1}{P} \frac{\mathrm{d} P}{\mathrm{~d} t}\right) t^{2}=\frac{1}{2}\left(\frac{\dot{P}}{P}\right) t^{2}$

star and the pulsation mode geometry, but for A and F stars the difference between Kepler and WASP amplitudes is of order 10 per cent. 

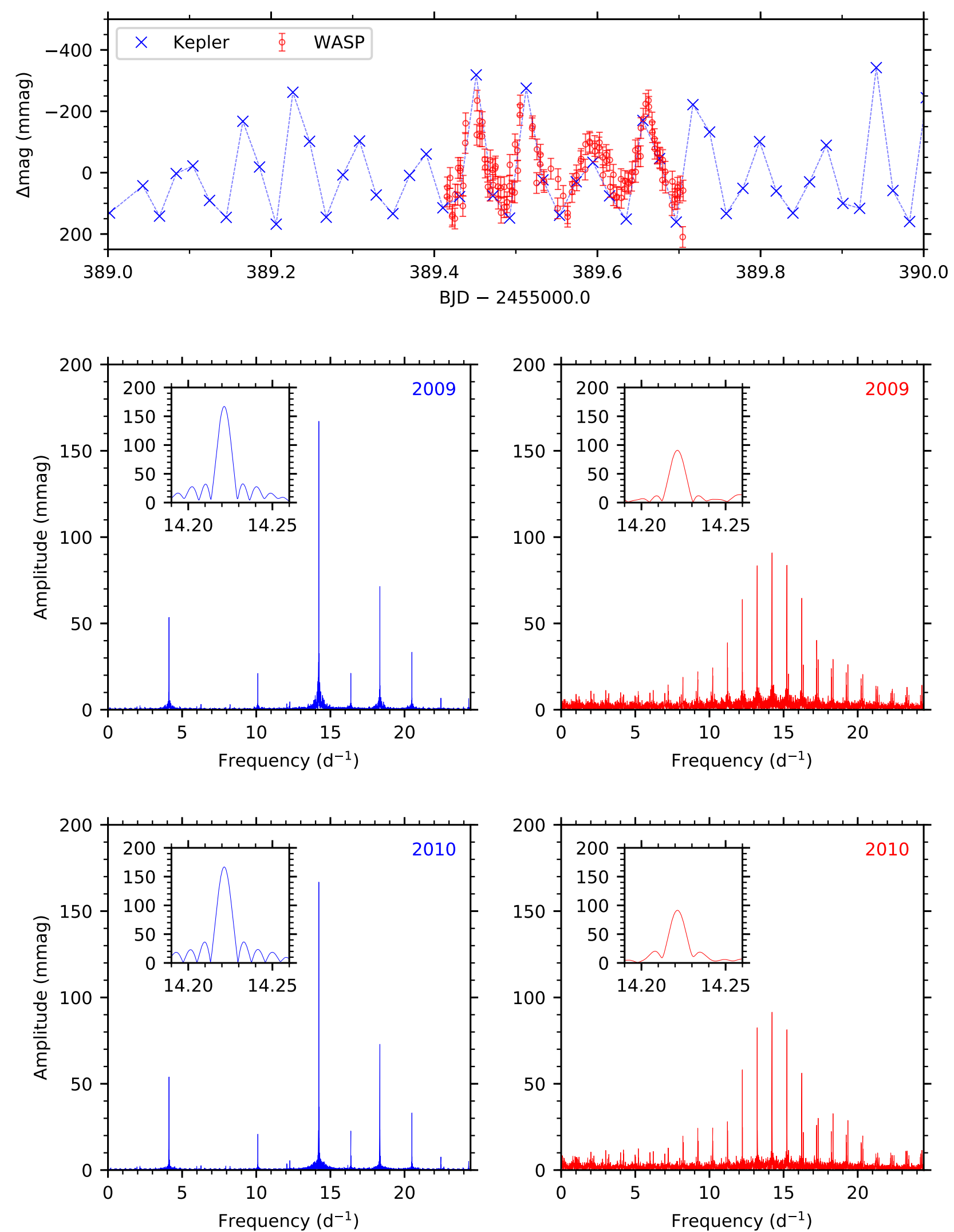

Figure 2. The top panel is a 1-d segment of the Kepler and WASP light curves of KIC 5950759 shown as blue crosses and red circles, respectively. The lower panels show amplitude spectra of KIC 5950759 labelled by their respective observing season, with the left panels denoting Kepler data in blue and the right panels denoting WASP data in red. In each panel, a sub-plot of the dominant pulsation mode frequency, $\nu_{1}$, is shown. The lower amplitudes of the pulsation modes in the WASP data is primarily caused by flux dilution in the relatively larger WASP pixels (see text for details). 

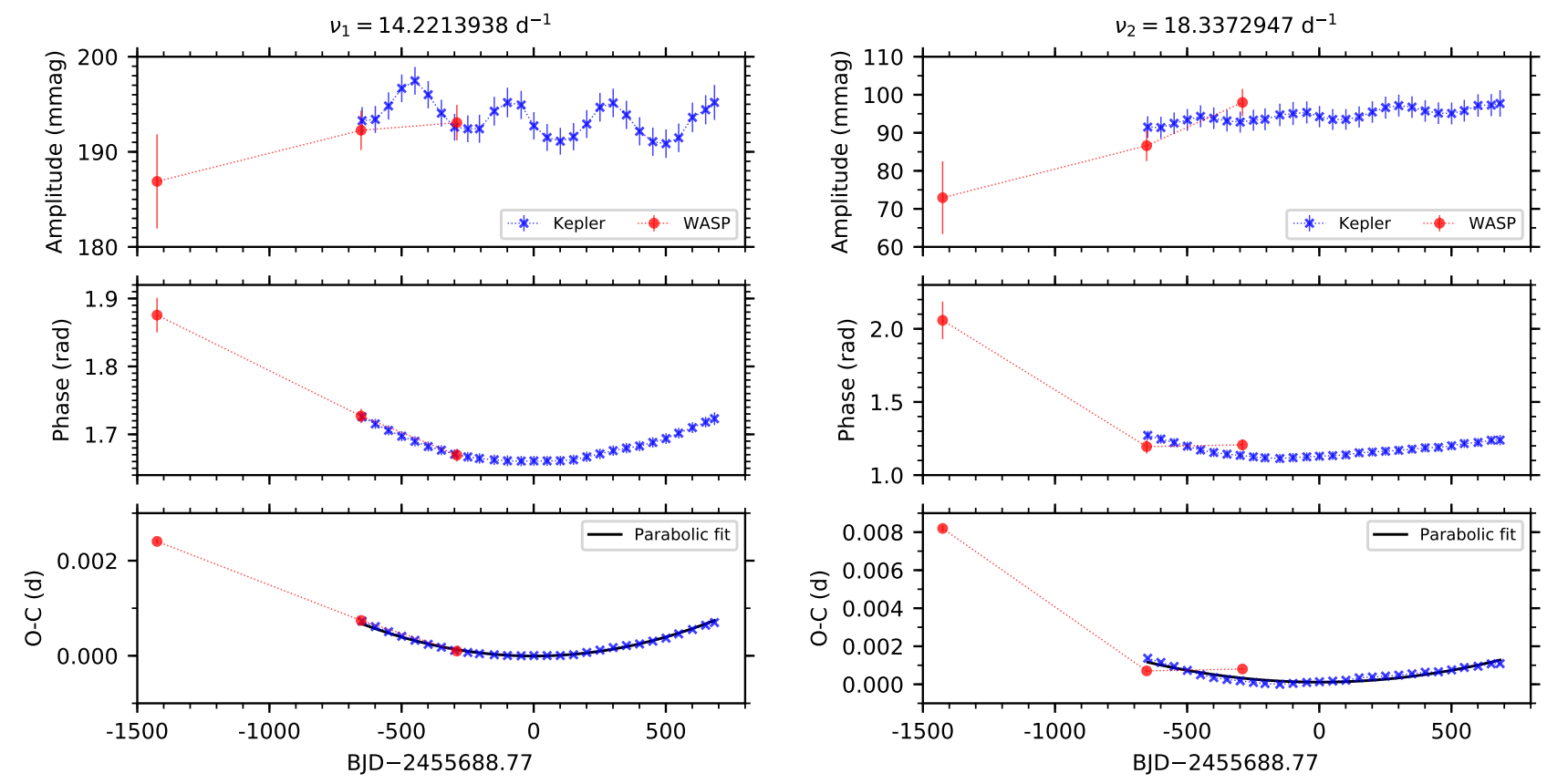

Figure 3. Inclusion of WASP data with Kepler data to study the long-term amplitude and phase modulation of the fundamental, $\nu_{1}$ (left), and first overtone, $\nu_{2}$ (right), radial modes in KIC 5950759. Top panels: amplitude modulation in KIC 5950759 using the available LC Kepler data (in bins of $150 \mathrm{~d}$ and $100-\mathrm{d}$ overlap) as blue crosses and the three WASP seasons as red circles, which have been corrected for their respective integration times, passbands and dilution (see text for details). Middle panels: phase modulation tracked at fixed frequency. Bottom panels: O-C diagram in which the parabolic fit reveals the linear rate of change in period for each pulsation mode. In all panels, the zero-point of the time scale has been taken as the centre of the LC Kepler data set (i.e. BJD 2455688.770). Note that the bottom two rows are equivalent.

where $\mathrm{O}-\mathrm{C}$ is the difference between the observed and calculated times of maxima in days, $t$ is the time span of the observations in days, and $P$ is the period of the signal in days, such that to convert the period change into its conventional units of $\mathrm{yr}^{-1}$ one uses the number of days in a year (i.e. 365.2422). Thus, Eq. (4) dictates that a linearly changing period produces a quadratic change in $\mathrm{O}-\mathrm{C}$ (Percy et al. 1980; Breger \& Pamyatnykh 1998; Sterken 2005).

In addition to studying the amplitude modulation of pulsation modes in KIC 5950759, the concurrent WASP and Kepler observations in 2009 and 2010 allow us the unique opportunity to probe its phase (i.e. frequency) modulation. Using the technique employed by Bowman et al. (2016), we calculate the pulsation phase using a linear least-squares fit to each of the 150-d bins of LC Kepler data, which is shown in the middle row of Fig. 3. Furthermore, to include the 2007 WASP data, as before we use the truncated 2009 and 2010 overlapping Kepler data to compare the pulsation phase during the concurrent 2009 and 2010 WASP seasons. Since we are assuming and fixing the pre-optimised pulsation mode frequencies of $\nu_{1}$ and $\nu_{2}$ (cf. Table 2), we perform an independent linear least-squares fit to each of the WASP and truncated Kepler data in 2009 and 2010. We compare the corresponding pulsation phases and find a constant phase offset of $0.54 \pm 0.01$ rad between the 2009 WASP and truncated 2009 Kepler data (and the same value between the 2010 WASP and truncated 2010 Kepler data). Such a phase offset is understandable given the difference in passband between the two instruments and because pulsation phases depend on wavelength ${ }^{5}$.

After correcting the 2007, 2009 and 2010 WASP phases for

${ }^{5}$ Pulsation mode phase differences is a method of mode identification for multi-colour time series photometric observations - see Watson (1988) and Heynderickx et al. (1994) for early applications of this technique. the passband phase offset value, we include them alongside the LC Kepler data of KIC 5950759 in the middle panels of Fig. 3. A clear parabolic change in pulsation phase is evident for both the fundamental $\left(\nu_{1}\right.$; middle-left panel of Fig. 3) and first overtone $\left(\nu_{2}\right.$; middle-right panel of Fig. 3$)$, which indicate a linearly increasing pulsation period. Under the reasonable assumption of negligible mass loss across a period of approximately $6 \mathrm{yr}$, this implies an increasing stellar radius in KIC 5950759. The observed phase modulation for each radial mode, $\nu_{1}$ and $\nu_{2}$, is converted using $\mathrm{O}-\mathrm{C}=\frac{\phi}{2 \pi \nu}$, with the resultant $\mathrm{O}-\mathrm{C}$ diagrams shown in the bottom-left and bottom-right panels of Fig. 3, respectively. To avoid adding unnecessary additional uncertainty, we fit only the Kepler data with Eq. (4) with $\left(\frac{1}{P} \frac{\mathrm{d} P}{\mathrm{~d} t}\right)$ as a free parameter. The fitted fractional rate of change in period values have units of $\mathrm{d}^{-1}$ since $t$ and $\mathrm{O}-\mathrm{C}$ are in units of $\mathrm{d}$, we converted them into the conventional unit of $\mathrm{yr}^{-1}$. This yielded best-fitting values of $(1.17 \pm 0.02) \times 10^{-6} \mathrm{yr}^{-1}$ and $(1.82 \pm 0.12) \times 10^{-6} \mathrm{yr}^{-1}$ for the fractional period changes of the fundamental and first overtone radial modes, respectively.

Contrary to stellar evolution theory, approximately an equal fraction of Population I $\delta$ Sct stars are observed to have increasing periods compared to those with decreasing periods in their radial modes (Breger 1990; Guzik \& Cox 1991). The typical linear period changes observed in $\delta$ Sct stars can be as large as $\dot{P} / P \simeq 10^{-7} \mathrm{yr}^{-1}$, which is at least an order of magnitude larger than those predicted by stellar evolution theory for main sequence $\delta$ Sct stars (Breger \& Pamyatnykh 1998). Similarly large period changes have also been observed in HADS stars (e.g. Walraven et al. 1992) and SX Phe stars (e.g. Murphy et al. 2013b). In KIC 5950759, we measure even larger period changes of order $10^{-6} \mathrm{yr}^{-1}$, which could be used to infer that this HADS star is in a relatively short- 
lived stage of stellar evolution, such as near or beyond the TAMS, if such variability is caused by evolution. The increasing period of the radial modes also implies that the radius of KIC 5950759 is increasing, which suggests a post-main sequence stage of evolution as opposed to the overall contraction phase. An order of magnitude estimate of the change in radius using the period-density relation for classical pulsators (see e.g. Stellingwerf 1979) and assuming a constant mass of $M=1.6 \mathrm{M}_{\odot}$ yields $\dot{R} \simeq 1 \mathrm{~km} \mathrm{yr}^{-1}$, but the uncertainties on this are substantial. Such a value is three orders of magnitude larger than expected from stellar evolution models. Hence it is unlikely that the observed period (and implied radius) changes are caused by evolution.

We note that the amplitude and phase modulation observed in KIC 5950759 are seemingly unique, because phase modulation in such stars appears as jumps rather than smooth variation over time (see e.g. Breger \& Pamyatnykh 1998; Boonyarak et al. 2011). On the other hand, few HADS stars have been studied using such high quality photometric time series as those provided by the Kepler mission. Since the observed phase modulation is much larger than predicted by evolution models, this indicates that the short-term evolution of some stars can be quite 'noisy', such that period changes measured over time spans of years and decades may be caused by physical processes that are not resolved by the large time steps taken in evolutionary calculations.

\section{SPECTROSCOPY}

KIC 5950759 is a relatively faint star within the nominal Kepler field of view with $V=13.516$ mag (Pigulski et al. 2009). To determine atmospheric parameters, and in particular an accurate effective temperature, $T_{\text {eff }}$, we obtained a spectrum on 2016-09-28 using the Intermediate Dispersion Spectrograph (IDS) mounted on the $2.5-\mathrm{m}$ Isaac Newton Telescope (INT) located at the Roque de los Muchachos Observatory on the island of La Palma, Spain. The spectrum was obtained using the R400V grating with a resolving power of $R \simeq 1500$ at $4500 \AA$. We reduced the data (including bias subtraction, flat fielding and wavelength calibration) using STARLINK ${ }^{6}$ software (Currie et al. 2014). We normalised the spectrum by fitting a spline through manually selected continuum points. A section of the normalised reduced IDS spectrum of KIC 5950759 is shown in Fig. 4. Spectral classification indicates that KIC 5950759 is a slow rotator from its narrow spectral lines, and is also a metal-poor A-type star given the lack of strong metal lines (Gray 2005; Gray \& Corbally 2009).

To analyse the spectrum of KIC 5950759 using a quantitative method and extract atmospheric parameters, we used the Grid Search in Stellar Parameters (GSSP) software (Tkachenko 2015). The GSSP code computes a grid of synthetic spectra across a range of values in effective temperature $\left(T_{\text {eff }}\right)$, surface gravity $(\log g)$, metallicity $([\mathrm{M} / \mathrm{H}])$, and projected surface rotational velocity $(v \sin i)$. The grid of synthetic spectra is computed using the SynthV LTE radiative transfer code (Tsymbal 1996) and atmospheric models computed from the LLMODELs code (Shulyak et al. 2004). In our analysis we fix the microturbutent and macroturbutent velocities to 2.0 and $0.0 \mathrm{~km} \mathrm{~s}^{-1}$, respectively, which are reasonable for stars of spectral type A (Gray 2005; Tkachenko et al. 2013; Niemczura et al. 2015). As mentioned previously, the low resolution of the observed spectrum makes it difficult to constrain

6 http://starlink.eao.hawaii.edu/starlink/ the surface gravity and rotation rate of KIC 5950759. Furthermore, for stars below approximately $8000 \mathrm{~K}$, the wings of Balmer lines are not sensitive to the surface gravity, which means that metal lines are typically used instead (Gray 2005; Smalley 2005). Given the resolving power of the IDS/INT instrument and the resultant resolution of the observed spectrum, rotational and instrumental broadening are indistinguishable for $v \sin i$ values below some $50 \mathrm{~km} \mathrm{~s}^{-1}$.

We shifted the observed spectrum to rest wavelengths and used the GSSP code to quantitatively compare it to each spectrum within the grid of synthetic spectra by means of minimising the corresponding $\chi^{2}$ function. After some initial tests, we confirmed that KIC 5950759 is a slow rotator, with $v \sin i \leq 50 \mathrm{~km} \mathrm{~s}^{-1}$, such that the inferred rotational broadening was smaller than the instrumental broadening. Therefore, we set the velocity broadening component to $0 \mathrm{~km} \mathrm{~s}^{-1}$ when using GSSP in our subsequent analysis. As a first step we use the $4250-5400 \AA$ wavelength range to constrain the effective temperature to $T_{\text {eff }}=7340 \pm 230 \mathrm{~K}$, which is primarily driven by the shape of the $H \gamma$ and $H \beta$ Balmer lines in this range. Given the low resolution of the spectrum and lack of strong metal lines, we are unable to constrain the surface gravity to better than $\log g=2.9 \pm 1.3 \mathrm{dex}$ and metallicity to $[\mathrm{M} / \mathrm{H}]=-1.18 \pm 0.30 \mathrm{dex}$. As a second step, we performed a second fitting scheme including a global continuum correction factor, which is assumed to be wavelength-independent and is computed in GSSP from the least-squares fit of the synthetic to the observed spectrum of the star. For the same wavelength regime of $4250-5400 \AA$, we constrained the effective temperature to $T_{\text {eff }}=7470 \pm 255 \mathrm{~K}$, the surface gravity to $\log g=3.6 \pm 1.2$ dex and the metallicity to $[\mathrm{M} / \mathrm{H}]=-0.65 \pm 0.45$ dex. These parameters are consistent with those from our previous analysis, and are provided in Table 1 .

As an additional confirmation of the sub-solar metallicity of KIC 5950759, we estimated the metallicity using the methodology of Smalley (1993). An estimate of the line blocking in the 4600$4700 \AA$ region using a model with $T_{\text {eff }}=7500 \mathrm{~K}$ and $\log g=3.5$ yielded a metallicity of $[\mathrm{M} / \mathrm{H}]=-1.1 \pm 0.4$, which is consistent with the sub-solar metallicity values obtained from GSSP. The high frequency of the fundamental radial mode in KIC 5950759, and the relatively large observed frequency ratio of 0.77554 also support that KIC 5950759 is a metal-poor star (see e.g. Petersen \& Christensen-Dalsgaard 1996; Poretti 2003; Murphy et al. 2013b; Daszyńska-Daszkiewicz et al. 2020).

\section{ESTIMATING THE LUMINOSITY FROM GAIA}

In their study of the period-luminosity (P-L) relation for $\delta$ Sct stars observed by Kepler with available Gaia parallaxes, Ziaali et al. (2019) include KIC 5950759 as an example of a star known to pulsate in the fundamental radial mode (see their figure 3). Interestingly, KIC 5950759 does not fall on the derived P-L relationship. Owing to the pulsation period being known to an extremely high precision, the discrepancy may be caused by an incorrect luminosity from an incorrect estimate of the interstellar reddening and/or distance. Murphy et al. (2019) calculated luminosities for a large sample of more than $2000 \delta$ Sct stars observed by the Kepler mission with available Gaia DR2 parallaxes (Gaia Collaboration et al. 2016, 2018), distances from Bailer-Jones et al. (2018) and dust maps from Green et al. (2018). From their ensemble analysis, Murphy et al. (2019) calculated a luminosity of $\log \left(L / \mathrm{L}_{\odot}\right)=1.017 \pm 0.038$ for KIC 5950759 assuming an effective temperature $T_{\text {eff }}=8070 \pm 280 \mathrm{~K}$.

The bolometric corrections of Murphy et al. (2019) were calculated based on parameters available from the Mathur et al. (2017) 


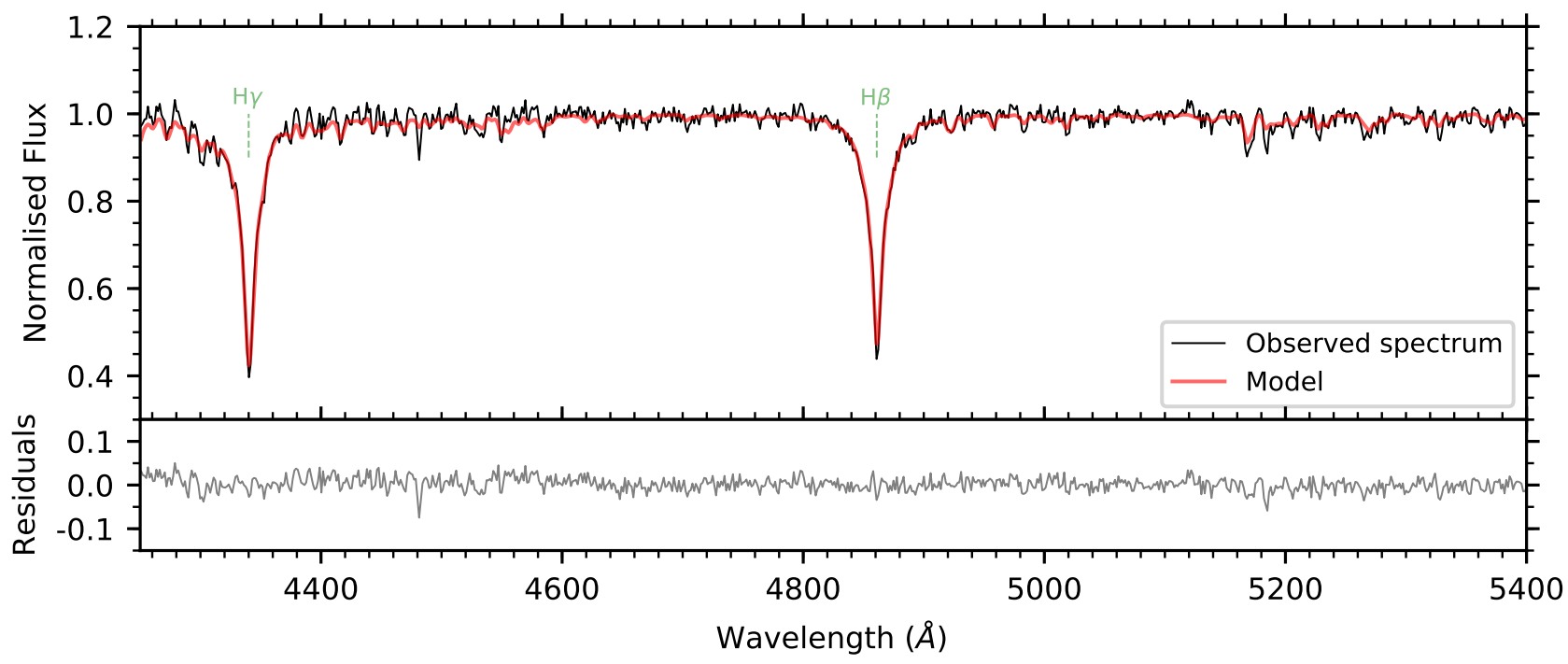

Figure 4. Top panel: observed spectrum of KIC 5950759 (black) and the best fitting model (without continuum correction) obtained using the GSSP software (Tkachenko 2015) in red. Bottom panel: residuals of model and observations.

stellar properties catalogue, which are somewhat consistent with those found in this work except for the metallicity, which we find to be sub-solar with $-1.18<[\mathrm{M} / \mathrm{H}]<-0.65 \mathrm{dex}$ (cf Table 1). This can have a large effect on the bolometric correction, so we have recalculated the luminosity using the updated astrometry from Gaia EDR3 (Gaia Collaboration et al. 2020), the effective temperatures, surface gravities and metallicities from our spectroscopic analysis, which yields $\log \left(L / \mathrm{L}_{\odot}\right)=1.064 \pm 0.045$. The fractional distance uncertainty for KIC 5950759 is $\sim 4$ per cent, whereas the fractional uncertainty on the $0.22 \mathrm{mag}$ extinction value is 16 per cent. Thus neither of these parameters is a likely source of the $\simeq 0.2$ mag difference between the calculated absolute magnitude of KIC 5950759 and the inferred P-L relation in Ziaali et al. (2019).

A potential cause of a star appearing too bright in the P-L diagram is binarity. The Gaia renormalised unit weight error (RUWE) has been interpreted as an indication of binarity, with RUWE $>2$ used to suggest binarity (Evans 2018; Rizzuto et al. 2018). In Gaia EDR3, the RUWE parameter for KIC 5950759 is 1.047 , so it is probably not a binary. Furthermore, after comparing the Gaia DR2 astrometric excess noise of 0.11 mas for KIC 5950759 to the average DR2 excess noise of 0.14 mas for the large sample of Kepler A and F stars from Murphy et al. (2019), we conclude that KIC 5950759 is unlikely to be a binary.

More importantly, McNamara (2011) found that corrections to the P-L relation were required for low-metallicity radial-mode pulsators. For stars of $[\mathrm{Fe} / \mathrm{H}] \simeq-1.0$, a correction of $0.2 \mathrm{mag}$ is required, such that a star is over-luminous by this amount compared to the luminosity predicted solely based on its dominant pulsation period (McNamara 2011). This correction would account for the location of KIC 5950759 being above the P-L relation found by Ziaali et al. (2019) for Kepler $\delta$ Sct stars. On the other hand, we cannot exclude an incorrect extinction for KIC 5950759 given its faint apparent brightness and larger inferred Gaia distance compared to typical A and F stars in the Kepler field.

Finally, we note that the large pulsation amplitude of KIC 5950759 causes substantial temperature, radius, and radial velocity variations during the pulsation cycle. Using equation 5 of Kjeldsen \& Bedding (1995), we estimate radial velocity variability of $\sim 100 \mathrm{~km} \mathrm{~s}^{-1}$ for measured brightness variations of $\pm 0.4 \mathrm{mag}$, given that in Kepler SC data the peak-to-peak light amplitude variations exceed $0.7 \mathrm{mag}$. We did not include the radius and temperature variations in the 10000 -iteration Monte Carlo simulation for our improved calculation of the luminosity of KIC 5950759 in this work. Yet, we did include a $1 \sigma$ apparent magnitude change of 0.14 mag calculated from the standard deviation of the Kepler brightness measurements.

\section{ASTEROSEISMIC MODELLING}

To ascertain the mass and evolutionary stage of KIC 5950759, we performed forward seismic modelling based on its observed pulsation mode frequencies, spectroscopic parameters and Gaia luminosity. Since Kepler data are of exceptionally high quality, the two dominant pulsation mode frequencies and their frequency ratio are known to a very high precision. The high amplitudes of the pulsation modes and their frequency ratio indicate that they are likely the fundamental and first overtone radial modes, so a sensible method to model KIC 5950759 is by using Petersen diagrams. The frequency and frequency ratio of radial modes depend primarily on the mass, age, evolutionary stage and metallicity. Thus, Petersen diagrams offer a useful diagnostic in constraining these parameters of radial pulsators (see e.g. Petersen 1973; Petersen \& Christensen-Dalsgaard 1996; Daszyńska-Daszkiewicz et al. 2020).

We calculated evolution models with the WARSAW-NewJERSEY code (see Pamyatnykh et al. 1998; Pamyatnykh 1999) using the OPAL opacity tables (Iglesias \& Rogers 1996) and a solar chemical mixture (Asplund et al. 2009) for various metallicity values. Rotation, or more specifically the mean effect of the centrifugal force, is included within the WARSAW-NEWJERSEY code, and solid-body rotation and constant global angular momentum during evolution are assumed. Convection in the stellar envelope is based on standard mixing length theory, and the convective flux is assumed to be constant throughout a pulsation cycle. This is more commonly known as the convective flux freezing approximation, which is robust if convection is not efficient, such as in the envelopes of early type 


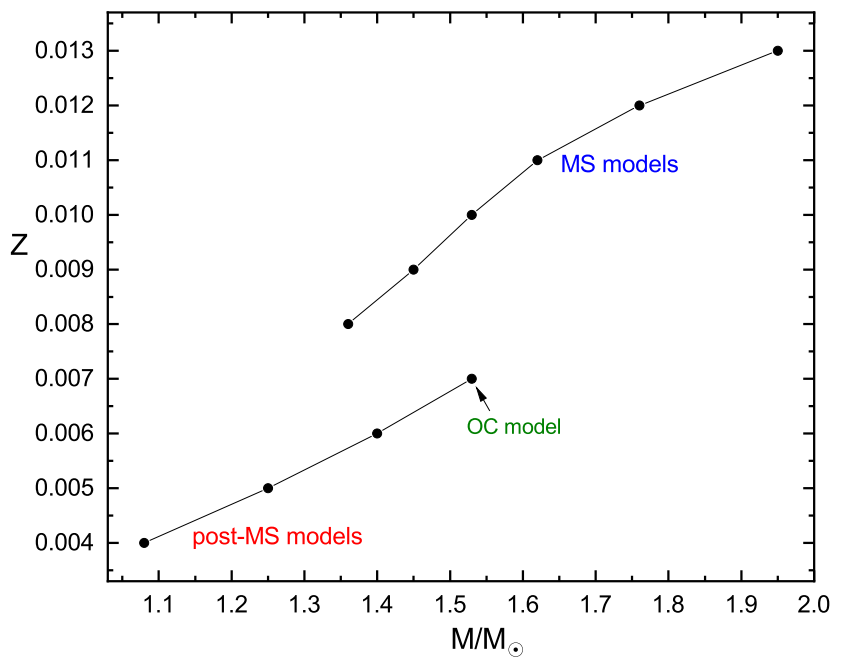

Figure 5. Models calculated across a range in metallicity which match the observed frequency ratio observed in KIC 5950759. The strong constraint of the observed frequency ratio creates two branches in terms of possible evolutionary phases (where $\mathrm{MS}=$ main sequence, $\mathrm{OC}=$ overall contraction phase, post-MS $=$ post main sequence) .

stars. Linear non-adiabatic pulsation models were calculated using the code of Dziembowski (1977).

As described in section 3, there are large uncertainties associated with determining the metallicity of a slowly rotating, highamplitude pulsating star with a low resolution spectrum. Therefore we chose to survey a range of models with different metallicities between $0.004 \leq Z \leq 0.013$ dex in steps of $0.001 \mathrm{dex}$, which is larger than the inferred $1 \sigma$ metallicity uncertainty from spectroscopy, and determine the best fitting mass and age. For each value of metallicity, we found the model that fits the dominant frequency $\left(\nu_{1}\right)$ identified as the fundamental radial mode, and closely matches the frequency identified as the first overtone radial mode $\left(\nu_{2}\right)$. Our results are shown in the mass-metallicity plane in Fig. 5. All the models in Fig. 5 match the observed frequencies $\nu_{1}$ and $\nu_{2}$, which demonstrates the strong mass-metallicity degeneracy. In general, fitting the two pulsation modes alone is unable to break the degeneracy between mass and age ${ }^{7}$. Hence one needs additional information, such as fitting a third mode or including spectroscopic constraints, to constrain the age of a star. Specifically, effective temperature and metallicity derived from spectroscopy and the luminosity estimated from Gaia are used to delimit the possible parameter space in the HR diagram.

As can be seen in Fig. 5, there are two branches of possible solutions for KIC 5950759: main-sequence models for which $Z \geq 0.008$ and post-main sequence models for which $0.004 \leq$ $Z<0.007$. For each model at a given metallicity shown in Fig. 5 , we provide the best-fitting mass, age, effective temperature, luminosity, theoretical frequency of the first overtone radial mode, and the frequency ratio of the fundamental and first overtone radial modes in Table 3. For each best-fitting model, we also calculate the normalised instability parameters $\eta\left(p_{1}\right)$ and $\eta\left(p_{2}\right)$ (as defined by Stellingwerf 1978; see also Daszyńska-Daszkiewicz et al. 2020),

7 Analogous to isochrones (lines of constant age), one can fit isopycnals or isopycnics (i.e. lines or surfaces of constant density) using frequency ratios of radial modes (see Hermans 2019) to serve as a proxy for stellar age in the HR diagram as opposed to using uncertain spectroscopic log $g$ values.
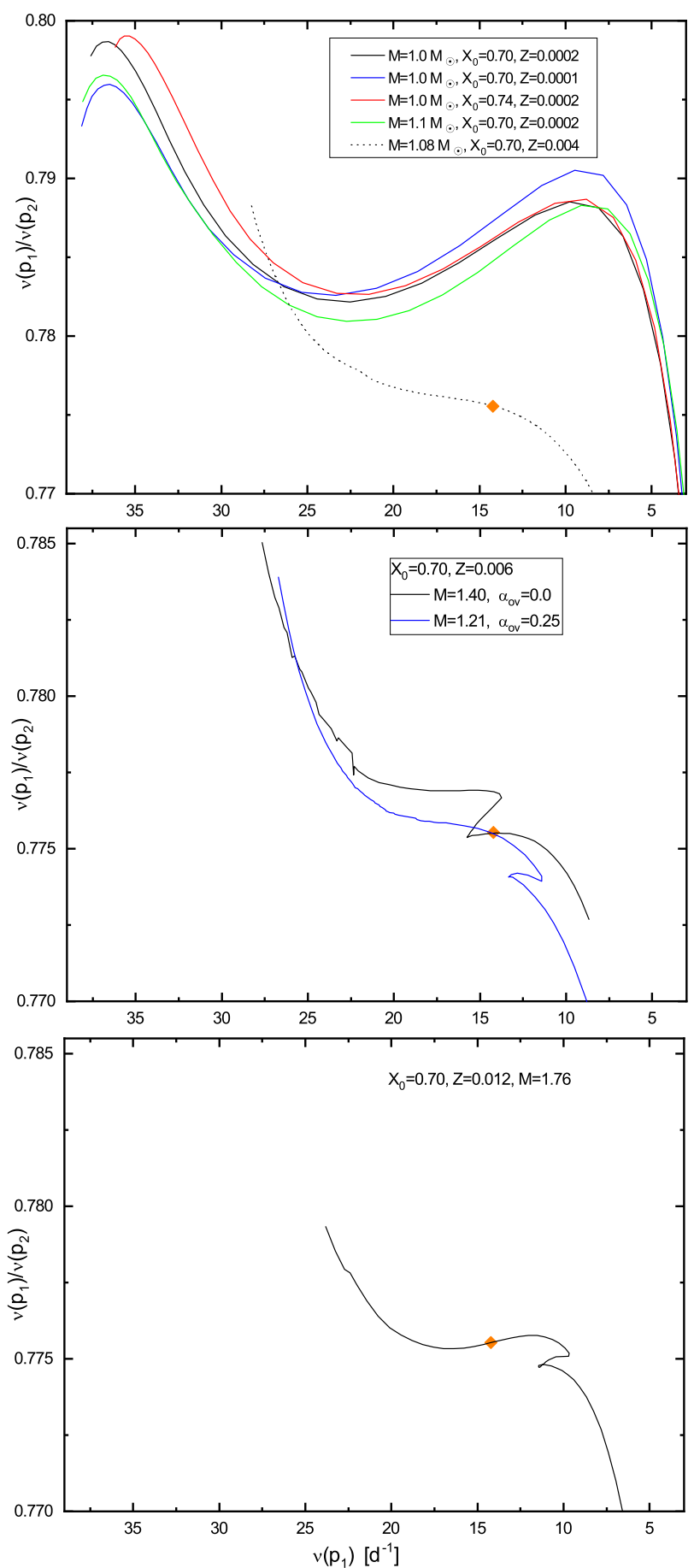

Figure 6. Petersen diagrams spanning from the zero-age main sequence to the immediate post-main sequence phase of evolution for various metallicity values in our grid. In each panel, the location of the best model is indicated by the orange diamond. The observed frequency ratio of $\nu_{1} / \nu_{2}$ for KIC 5950759 clearly excludes low metallicities (i.e. $Z<0.003$ ).

for which positive values indicate that a pulsation mode is excited (i.e. unstable) and negative values indicate it is not (i.e. stable).

However, not all models shown in Fig. 5 lie within $3 \sigma$ of the spectroscopic constraints on the effective temperature and Gaia luminosity for KIC 5950759. To demonstrate the importance of these additional constraints in forward seismic modelling, we show Petersen diagrams for different parameter combinations in Fig. 6. 
Table 3. The parameters of the models that reproduce exactly the observed dominant frequency $\nu_{1}$ as the radial fundamental mode $\left(\ell=0, p_{1}\right)$ and closely match the observed frequency $\nu_{2}$ as the radial first overtone $\left(\ell=0, p_{2}\right)$. All models have an initial hydrogen abundance of $X_{0}=0.70$, mixing length parameter $\alpha_{\mathrm{MLT}}=0.1$ and an initial rotational velocity $v_{\text {rot }}=10 \mathrm{~km} \mathrm{~s}^{-1}$. Columns are metallicity $(Z)$, the convective core overshooting $\left(\alpha_{\mathrm{ov}}\right)$, mass $(M)$, phase of evolution (where OC denotes the overall contraction phase), age, effective temperature $\left(\log T_{\text {eff }}\right), \operatorname{luminosity}(\log L / L \odot)$, the theoretical frequency of the radial first overtone $\nu\left(p_{2}\right)$, the frequency ratio of the first two radial modes and their normalised instability parameters $\eta\left(p_{1}\right)$ and $\eta\left(p_{2}\right)$, and the theoretically predicted fractional period change of the fundamental radial mode.

\begin{tabular}{|c|c|c|c|c|c|c|c|c|c|c|c|}
\hline$Z$ & $\alpha_{\mathrm{ov}}$ & $\begin{array}{c}M \\
\left(\mathrm{M}_{\odot}\right)\end{array}$ & phase & $\begin{array}{l}\text { age } \\
(\mathrm{Gyr})\end{array}$ & $\log T_{\text {eff }}$ & $\log L / \mathrm{L}_{\odot}$ & $\begin{array}{l}\nu\left(p_{2}\right) \\
\left(\mathrm{d}^{-1}\right)\end{array}$ & $\nu\left(p_{1}\right) / \nu\left(p_{2}\right)$ & $\eta\left(p_{1}\right)$ & $\eta\left(p_{2}\right)$ & $\begin{array}{c}\dot{P} / P \\
\left(\mathrm{yr}^{-1}\right)\end{array}$ \\
\hline 0.004 & 0.0 & 1.08 & post-MS & 3.66 & 3.8285 & 0.743 & 18.33686 & 0.77556 & +0.079 & +0.036 & $+8.5 \times 10^{-10}$ \\
\hline 0.005 & 0.0 & 1.25 & post-MS & 2.25 & 3.8652 & 0.932 & 18.33704 & 0.77556 & +0.068 & +0.066 & $+1.2 \times 10^{-9}$ \\
\hline 0.006 & 0.0 & 1.40 & post-MS & 1.62 & 3.8919 & 1.071 & 18.33817 & 0.77551 & -0.029 & +0.015 & $+1.0 \times 10^{-9}$ \\
\hline 0.006 & 0.25 & 1.21 & MS & 2.60 & 3.8195 & 0.737 & 18.33857 & 0.77549 & +0.070 & +0.024 & $+6.1 \times 10^{-10}$ \\
\hline 0.007 & 0.0 & 1.53 & OC & 1.31 & 3.9099 & 1.167 & 18.33867 & 0.77549 & -0.143 & -0.071 & $-2.3 \times 10^{-8}$ \\
\hline 0.008 & 0.0 & 1.36 & MS & 1.73 & 3.8359 & 0.835 & 18.33685 & 0.77556 & +0.082 & +0.045 & $+7.7 \times 10^{-10}$ \\
\hline 0.009 & 0.0 & 1.45 & MS & 1.42 & 3.8510 & 0.914 & 18.33668 & 0.77557 & +0.079 & +0.058 & $+8.2 \times 10^{-10}$ \\
\hline 0.010 & 0.0 & 1.53 & MS & 1.21 & 3.8637 & 0.979 & 18.33759 & 0.77553 & +0.059 & +0.058 & $+8.8 \times 10^{-10}$ \\
\hline 0.011 & 0.0 & 1.62 & MS & 1.02 & 3.8775 & 1.051 & 18.33797 & 0.77552 & +0.016 & +0.038 & $+9.4 \times 10^{-10}$ \\
\hline 0.012 & 0.0 & 1.76 & MS & 0.78 & 3.9014 & 1.170 & 18.33771 & 0.77555 & -0.104 & -0.048 & $+1.1 \times 10^{-9}$ \\
\hline 0.013 & 0.0 & 1.95 & MS & 0.56 & 3.9324 & 1.323 & 18.33985 & 0.77544 & -0.326 & -0.256 & $+1.3 \times 10^{-9}$ \\
\hline
\end{tabular}
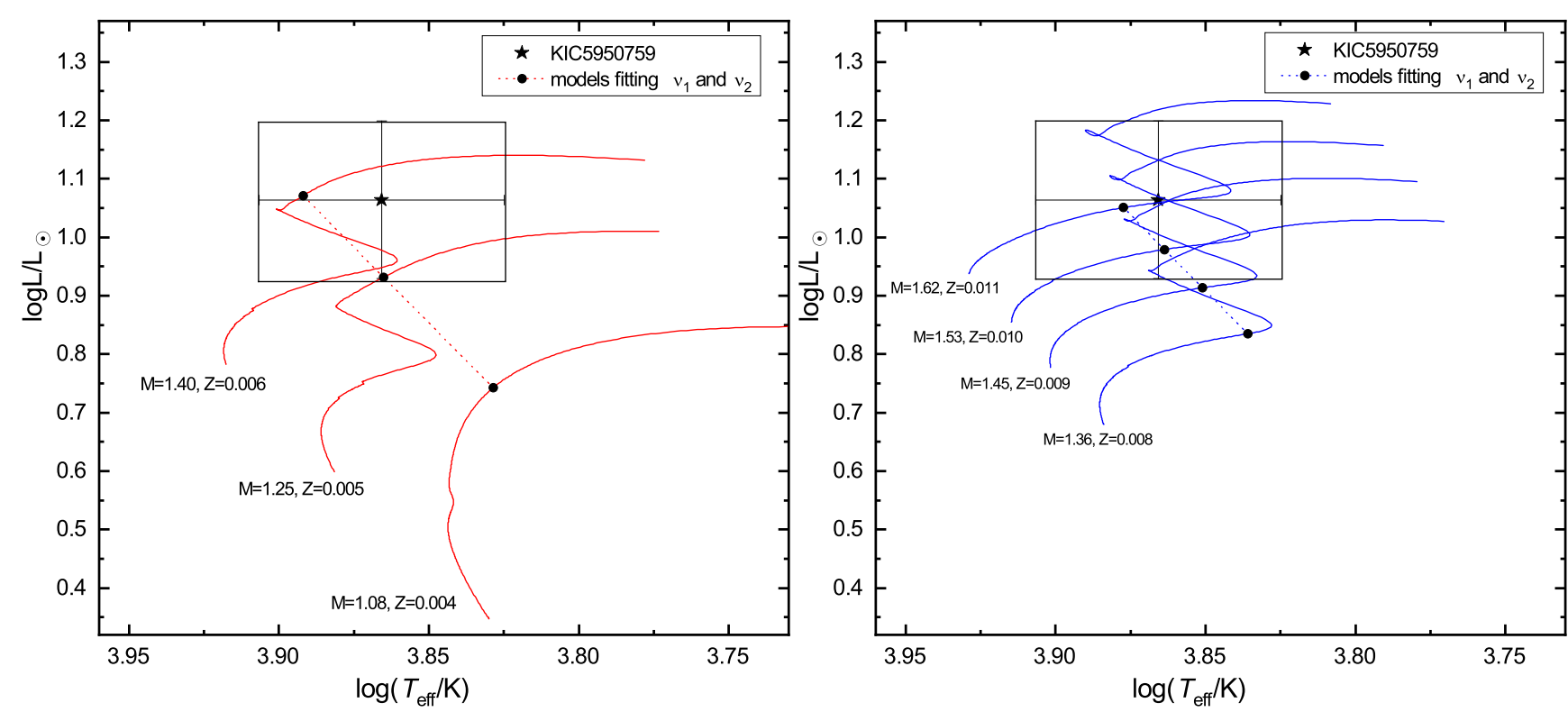

Figure 7. HR diagram with the $3 \sigma T_{\text {eff }}-\log L$ error box of KIC 5950759 and evolutionary tracks computed for various masses and metallicities at the adopted values of an initial hydrogen abundance $X_{0}=0.7$, the mixing length parameter $\alpha_{\mathrm{MLT}}=0.1$ and no convective core overshooting. Seismic models that best reproduce the two dominant frequencies as the fundamental and first overtone radial modes are marked with dots. In the left panel, the best-fitting seismic models are in the post-main sequence phase of evolution and in the right panel they are in main sequence phase of evolution.

In the top panel, Petersen diagrams for extremely metal-poor stars are shown as coloured lines; none of which match the observed frequency ratio of KIC 5950759. On the contrary, the observed frequency ratio excludes the possibility of KIC 5950759 having $Z<$ 0.003 , with the lowest value in our grid that matches the frequency ratio being $Z=0.004$. At the other end of the metallicity scale are main sequence models with higher masses and younger ages that match the observed frequency ratio of KIC 5950759. We show an example of a near-solar metallicity (i.e. $Z=0.012$ ) Petersen diagram in the bottom panel of Fig. 6. Therefore, the metallicity of KIC 5950759 must lie within the range of $0.004 \leq Z \leq 0.013$, if one only considers the fit using only the fundamental and first overtone radial mode frequencies. In Fig. 7, we show the position of KIC 5950759 with its $3 \sigma$ error box and seismic models with the corresponding evolutionary tracks for several metallicities. In the left panel we marked seismic models in the post-main sequence phase of evolution and in the right panel seismic models in main sequence phase of evolution. The location of KIC 5950759 in the HR diagram combined with the best fitting frequency ratio of its fundamental and first overtone radial modes indicates a relatively low mass star $\left(M \leq 1.6 \mathrm{M}_{\odot}\right)$ with sub-solar metallicity near the overall contraction phase and the TAMS.

We also explored including convective core overshooting as a parameter in our models. The WARSAW-NEWJERSEY code uses a two parameter prescription introduced by Dziembowski \& Pamyatnykh (2008), which allows for a non-zero gradient of the hydrogen abundance inside the partly mixed region above the convective core. An example of the effect of overshooting with the efficiency parameter 
$\alpha_{\mathrm{ov}}=0.25$ for $Z=0.006$ is shown in the middle panel of Fig. 6 . Increasing the amount of near-core mixing provides the convective core with additional hydrogen available for nuclear burning during the main sequence. This extends the main sequence lifetime of the star and moves the location of the TAMS and blue hook to larger radii and hence higher luminosities in the HR diagram. Consequently, it also moves the TAMS to smaller frequency ratios in the Petersen diagram. Therefore, for $Z=0.006$, as shown in the middle panel of Fig. 6 and listed in Table 3, the best fitting model with $Z=0.006$ and $\alpha_{\mathrm{ov}}=0.00$ is a post-main sequence star, and with $Z=0.006$ and $\alpha_{\mathrm{ov}}=0.25$ it is a main sequence star.

A major advantage of using non-adiabatic pulsation models is that we are also able to determine if modes are excited. As can be seen in Table 3, the normalised instability parameters, $\eta\left(p_{1}\right)$ and $\eta\left(p_{2}\right)$, are negative for $Z \geq 0.012$, which means we can exclude relatively high mass (i.e. $M \geq 1.7 \mathrm{M}_{\odot}$ ) main sequence models as we do not expect the fundamental and first overtone radial modes to be excited. Furthermore, the best fitting model for $Z=0.007$ also has negative $\eta\left(p_{1}\right)$ and $\eta\left(p_{2}\right)$ values, which means that we can exclude the overall contraction phase. Combining the requirement for positive normalised instability parameters with the $3 \sigma$ confidence intervals for $T_{\text {eff }}$ and $\log L$ for KIC 5950759, the best fitting models are those with metallicity values approximately in the two ranges of $Z \in(0.005,0.006) \cup(0.010,0.011)$. However, our bestfitting models consistently place KIC 5950759 near to the TAMS ${ }^{8}$. Given this result and the limitations and uncertainties associated with the determination of $T_{\mathrm{eff}}, v \sin i$, and $Z$ for KIC 5950759, it is highly desirable that high-resolution spectra of KIC 5950759 be obtained. Preferably spectra sampling the pulsation cycle of $\nu_{1}$ should be obtained. This will allow a more accurate determination of the metallicity and effective temperature given that the large amplitude pulsations of KIC 5950759 are likely causing significant line broadening which cannot be taken into account in the analysis of only a single spectrum.

Motivated by the large observed values for the fractional period change in the fundamental and first overtone radial modes, we also checked the theoretically predicted values within our models for agreement. For completeness we extracted the fractional period change of the fundamental radial mode of the best-fitting model at each metallicity value in our grid. These values are provided in the last column of Table 3. The values are similar for the first overtone radial mode, and also comparable to those calculated by Breger \& Pamyatnykh (1998). Yet, all predicted values are considerably smaller than the fractional period change observed in KIC 5950759 by at least two orders of magnitude. It is well established that $\delta$ Sct stars (both Population I and II) have observed period changes much larger than predicted by evolutionary models (Rodríguez et al. 1995; Rodríguez \& Breger 2001; Breger \& Pamyatnykh 1998; Bowman et al. 2016). Therefore, we conclude that the (common) observed period changes in $\delta$ Sct pulsators are not the result of stellar evolution, but are likely connected to the inherent non-linear excitation mechanism of high-amplitude pulsation modes, and the interactions of modes causing modulated amplitudes and frequencies over time scales of years and decades (e.g. resonant mode coupling; Dziembowski \& Krolikowska 1985; Moskalik 1985; Breger \& Montgomery 2014; Bowman et al. 2016). Therefore, our results

\footnotetext{
8 Note that the TAMS as defined by Iben (1967) is before the overall contraction phase and blue hook in the HR diagram, such that $X_{c} \simeq 0.05$. Whereas other studies, such as Dotter (2016), define the TAMS as when the core hydrogen content (numerically) reaches zero.
}

demonstrate the need for future studies to move beyond linear asteroseismology, and include additional constraints based on pulsation amplitudes and non-linear effects at work in $\delta$ Sct stars.

\section{CONCLUSIONS}

In this work we have performed photometric, spectroscopic and modelling analyses of the high-amplitude $\delta$ Sct star KIC 5950759. Originally identified as having unique properties owing to its long term and large fractional period change in its fundamental radial and first overtone radial modes by Bowman (2016), our detailed frequency analysis of the Kepler mission photometry of KIC 5950759 reveals additional non-radial pulsation modes. We extend the 4-yr time span of the Kepler mission using WASP data, and demonstrate that KIC 5950759 exhibits long-term amplitude and phase modulation in its two dominant radial modes. The measured fractional linearly increasing periods of the fundamental and first overtone radial mode are of order $10^{-6} \mathrm{yr}^{-1}$, which are at least two orders of magnitude larger than predicted for evolutionary changes in main sequence and post-main sequence stars. Given such a large discrepancy is not supported by stellar evolution models, we conclude that non-linear mode interaction is an explanation for the time-dependent phenomena observed in KIC 5950759, as predicted by Dziembowski \& Krolikowska (1985) and Moskalik (1985).

We obtained and analysed low-resolution spectroscopy of KIC 5950759 using the GSSP software package (Tkachenko 2015). The determined effective temperature places KIC 5950759 within the predicted and observed instability region for $\delta$ Sct stars (see Murphy et al. 2019), albeit somewhat closer to the red edge but nonetheless within the typical parameter space of high-amplitude $\delta$ Sct stars (Petersen \& Christensen-Dalsgaard 1996; McNamara 2000a). On the other hand, our spectroscopic analysis was unable to accurately constrain the rotational velocity of KIC 5950759 owing to the total broadening being smaller than the instrumental broadening. Also, the low resolution of the spectrum increased the uncertainties associated with determining the surface gravity and metallicity of KIC 5950759, but the best-fitting values provided by GSSP are given in Table 1. We compared previous determinations of the luminosity of KIC 5950759 by Murphy et al. (2019) to new calculations based on Gaia EDR3 parameters (Gaia Collaboration et al. 2020) and conclude that KIC 5950759 is a single star as opposed to a binary.

Using the observed pulsation mode frequencies from the $\mathrm{Ke}$ pler mission data, the spectroscopic constraints on $T_{\text {eff }}$ and metallicity, the Gaia luminosity, and the normalised instability parameters for the fundamental and first overtone, we determined the most likely masses and ages of KIC 5950759 using forward seismic modelling based on Petersen diagrams. Our results are summarised in Table 3. We find that the best-fitting seismic models considering all the available constraints exist within two branches of the subsolar metallicity regime (i.e. $Z \in(0.005,0.006) \cup(0.010,0.011)$ ). Our spectroscopic metallicity favours the lower-metallicity range in our models (i.e. $Z \in(0.005,0.006)$, which further strengthens the conclusion that KIC 5950759 is near the TAMS. Multi-epoch high resolution spectroscopy that effectively samples the dominant pulsation cycle of KIC 5950759 is needed to accurately determine its atmospheric parameters. Large amplitude pulsations induce significant changes in the effective temperature and radius of a star, and such behaviour is not captured within our analysis of a single low resolution spectrum.

Most interestingly, the relatively large increasing period 
changes in the fundamental and first overtone radial modes combined with our modelling results indicates that such an observed period change is too large to be caused directly by stellar evolution. This is in agreement with observations of $\delta$ Sct stars from the ground (see e.g. Breger \& Pamyatnykh 1998). Our modelling results indicate that KIC 5950759 is close to, if not just past, the TAMS. Despite this being the parameter space where period changes caused by evolution are predicted to be maximal, the observed amplitude and phase modulation of KIC 5950759 is too large to be directly caused by evolution. Given that the high-amplitude pulsations of HADS stars are sufficient to drive a periodic change in the stellar radius of order a factor two every $\simeq 2 \mathrm{hr}$, we posit that the observed amplitude and phase modulation is the manifestation of the star's structure responding to a highly non-linear driving mechanism. Post-main sequence $\delta$ Sct or post-main sequence HADS stars are not uncommon, but since HADS stars themselves make up only a small minority of all A and F stars, this only lends further evidence that KIC 5950759 is a unique object within all the known $\delta$ Sct stars observed by the Kepler mission.

In the future, we expect ongoing space photometry missions, such as TESS (Ricker et al. 2015), to find additional interesting HADS stars in, or close to, relatively short lived stages of stellar evolution. Currently, it is difficult to study the evolutionary changes in $\delta$ Sct stars from their pulsations in real time as they occur on such long time scales compared to observations. Moreover, non-linear mode interactions causing significant amplitude and phase modulation seemingly dominate their temporal behaviour (Breger 2000c; Breger \& Montgomery 2014; Bowman \& Kurtz 2014; Bowman et al. 2016). However, for relatively short-lived phases of evolution, such as the overall contraction phase and the immediate post-main sequence, with a large enough sample of $\delta$ Sct stars observed by TESS, we should expect to increase this sample size to a nonnegligible number of candidates. Ultimately, a large enough sample of pulsating stars with similar temporal properties to KIC 5950759 will allow us to probe non-linear pulsations and their effect on stellar structure in real time across a range of stellar masses and metallicities, and determine why such large period changes are present in so many $\delta$ Sct stars, but not in all.

\section{ACKNOWLEDGEMENTS}

DMB gratefully acknowledges a senior postdoctoral fellowship from the Research Foundation Flanders (FWO) with grant agreement No. 1286521 N. JDD acknowledges support from the Polish National Science Center (NCN), grant no. 2018/29/B/ST9/02803. DLH acknowledges the Science and Technology Facilities Council (STFC) via grant ST/M000877/1. SJM is a DECRA fellow supported by the Australian Research Council, grant number DE180101104. This work was supported by the Australian Research Council, an Australian Government Research Training Program (RTP) scholarship, and the Danish National Research Foundation (Grant DNRF106) through its funding for the Stellar Astrophysics Centre (SAC). The research leading to these results has received funding from the European Research Council (ERC) under the European Union's Horizon 2020 research and innovation programme (grant agreement No. 670519: MAMSIE), from the KU Leuven Research Council (grant C16/18/005: PARADISE), from the Research Foundation Flanders (FWO) under grant agreements G0H5416N (ERC Runner Up Project), as well as from the BELgian federal Science Policy Office (BELSPO) through PRODEX grant PLATO.

The Kepler data presented in this paper were obtained from the Mikulski Archive for Space Telescopes (MAST) at the Space Telescope Science Institute (STScI), which is operated by the Association of Universities for Research in Astronomy, Inc., under NASA contract NAS5-26555. Support to MAST for these data is provided by the NASA Office of Space Science via grant NAG57584 and by other grants and contracts. Funding for the Kepler/K2 mission was provided by NASA's Science Mission Directorate. The Gaia data in this paper come from the European Space Agency mission Gaia, processed by the Gaia Data Processing and Analysis Consortium (DPAC). Funding for the DPAC has been provided by national institutions, in particular the institutions participating in the Gaia Multilateral Agreement. The WASP project is funded and operated by Queen's University Belfast, the Universities of Keele, St. Andrews and Leicester, the Open University, the Isaac Newton Group, the Instituto de Astrofísica de Canarias, the South African Astronomical Observatory, and by STFC.

This research made use of the Starlink software (Currie et al. 2014), which is currently supported by the East Asian Observatory. This research has made use of the SIMBAD database, operated at CDS, Strasbourg, France; the SAO/NASA Astrophysics Data System; and the VizieR catalogue access tool, CDS, Strasbourg, France. This research has made use of community-developed core PYTHON packages for astronomy (ASTROPY; Astropy Collaboration et al. 2013, 2018), the PYTHON library for publication quality graphics (MATPLOTLIB; Hunter 2007), Numpy (van der Walt et al. 2011), and SciPy (Virtanen et al. 2020).

\section{DATA AVAILABILITY}

The Kepler data underlying this article are available from the MAST archive (http://archive.stsci.edu/kepler/). All other data underlying this article are available from the corresponding author upon reasonable request.

\section{REFERENCES}

Aerts C., 2021, Reviews of Modern Physics, 93, 015001

Aerts C., Christensen-Dalsgaard J., Kurtz D. W., 2010, Asteroseismology. Springer

Antoci V., et al., 2014, ApJ, 796, 118

Antoci V., et al., 2019, MNRAS, 490, 4040

Asplund M., Grevesse N., Sauval A. J., Scott P., 2009, ARA\&A, 47, 481

Astropy Collaboration et al., 2013, A\&A, 558, A33

Astropy Collaboration et al., 2018, AJ, 156, 123

Bailer-Jones C. A. L., Rybizki J., Fouesneau M., Mantelet G., Andrae R., 2018, AJ, 156, 58

Balona L. A., 2016, MNRAS, 459, 1097

Balona L. A., Dziembowski W. A., 2011, MNRAS, 417, 591

Balona L. A., Nemec J. M., 2012, MNRAS, 426, 2413

Balona L. A., Daszyńska-Daszkiewicz J., Pamyatnykh A. A., 2015, MNRAS, 452, 3073

Boonyarak C., Fu J.-N., Khokhuntod P., Jiang S.-Y., 2011, Ap\&SS, 333, 125

Borucki W. J., et al., 2010, Science, 327, 977

Bowman D. M., 2016, PhD thesis, Jeremiah Horrocks Institute, University of Central Lancashire, Preston, UK.

Bowman D. M., 2017, Amplitude Modulation of Pulsation Modes in Delta Scuti Stars. Springer International Publishing, doi:10.1007/978-3-31966649-5

Bowman D. M., Kurtz D. W., 2014, MNRAS, 444, 1909

Bowman D. M., Kurtz D. W., 2018, MNRAS, 476, 3169

Bowman D. M., Holdsworth D. L., Kurtz D. W., 2015, MNRAS, 449, 1004

Bowman D. M., Kurtz D. W., Breger M., Murphy S. J., Holdsworth D. L., 2016, MNRAS, 460, 1970 
Breger M., 1990, in Cacciari C., Clementini G., eds, Astronomical Society of the Pacific Conference Series Vol. 11, Confrontation Between Stellar Pulsation and Evolution. pp 263-273

Breger M., 2000a, in Szabados L., Kurtz D., eds, Astronomical Society of the Pacific Conference Series Vol. 203, IAU Colloq. 176: The Impact of Large-Scale Surveys on Pulsating Star Research. pp 421-425

Breger M., 2000b, in Breger M., Montgomery M., eds, Astronomical Society of the Pacific Conference Series Vol. 210, Delta Scuti and Related Stars. p. 3

Breger M., 2000c, MNRAS, 313, 129

Breger M., Montgomery M. H., 2014, ApJ, 783, 89

Breger M., Pamyatnykh A. A., 1998, A\&A, 332, 958

Breger M., et al., 1993, A\&A, 271, 482

Breger M., et al., 2011, MNRAS, 414, 1721

Brickhill A. J., 1992, MNRAS, 259, 519

Brown T. M., Latham D. W., Everett M. E., Esquerdo G. A., 2011, AJ, 142, 112

Butters O. W., et al., 2010, A\&A, 520, L10

Coates D. W., Halprin L., Thompson K., 1982, MNRAS, 199, 135

Currie M. J., Berry D. S., Jenness T., Gibb A. G., Bell G. S., Draper P. W., 2014, in Manset N., Forshay P., eds, Astronomical Society of the Pacific Conference Series Vol. 485, Astronomical Data Analysis Software and Systems XXIII. p. 391

Daszyńska-Daszkiewicz J., Pamyatnykh A. A., Walczak P., Szewczuk W., 2020, MNRAS, 499, 3034

Degroote P., et al., 2009, A\&A, 506, 111

Derekas A., et al., 2009, MNRAS, 394, 995

Dotter A., 2016, ApJS, 222, 8

Dupret M. A., Grigahcène A., Garrido R., Gabriel M., Scuflaire R., 2005, A\&A, 435, 927

Dziembowski W., 1977, Acta Astronomica, 27, 95

Dziembowski W., Krolikowska M., 1985, Acta Astronomica, 35, 5

Dziembowski W. A., Pamyatnykh A. A., 2008, MNRAS, 385, 2061

Evans D. F., 2018, Research Notes of the American Astronomical Society, 2, 20

Frolov M. S., Irkaev B. N., 1984, Information Bulletin on Variable Stars, 2462

Gaia Collaboration et al., 2016, A\&A, 595, A1

Gaia Collaboration et al., 2018, A\&A, 616, A1

Gaia Collaboration Brown A. G. A., Vallenari A., Prusti T., de Bruijne J. H. J., Babusiaux C., Biermann M., 2020, arXiv e-prints, p. arXiv:2012.01533

Goodricke J., 1783, Philosophical Transactions of the Royal Society of London Series I, 73, 474

Gray D. F., 2005, The Observation and Analysis of Stellar Photospheres, third edn. Cambridge University Press

Gray R. O., Corbally J. C., 2009, Stellar Spectral Classification. Princeton Series in Astrophysics

Green G. M., et al., 2018, MNRAS, 478, 651

Guzik J. A., Cox A. N., 1991, Delta Scuti Star Newsletter, 3, 6

Hermans J., 2019, Master's thesis, Institute of Astronomy, KU Leuven, Belgium

Heynderickx D., Waelkens C., Smeyers P., 1994, A\&AS, 105, 447

Holdsworth D. L., 2015, PhD thesis, Keele University, UK

Huber D., et al., 2014, ApJS, 211, 2

Hunter J. D., 2007, Computing in Science and Engineering, 9, 90

Iben Jr. I., 1967, ARA\&A, 5, 571

Iglesias C. A., Rogers F. J., 1996, ApJ, 464, 943

Jayasinghe T., et al., 2020, MNRAS, 493, 4186

Kippenhahn R., Weigert A., Weiss A., 2012, Stellar Structure and Evolution. Springer, doi:10.1007/978-3-642-30304-3

Kjeldsen H., Bedding T. R., 1995, A\&A, 293, 87

Kurtz D. W., 1985, MNRAS, 213, 773

Kurtz D. W., Shibahashi H., Murphy S. J., Bedding T. R., Bowman D. M., 2015, MNRAS, 450, 3015

Lares-Martiz M., Garrido R., Pascual-Granado J., 2020, MNRAS, 498, 1194

Lee Y.-H., Kim S. S., Shin J., Lee J., Jin H., 2008, PASJ, 60, 551

Loumos G. L., Deeming T. J., 1978, Ap\&SS, 56, 285
Maeder A., 2009, Physics, Formation and Evolution of Rotating Stars. Springer, doi:10.1007/978-3-540-76949-1

Maeder A., Meynet G., 2000, ARA\&A, 38, 143

Mathur S., et al., 2017, ApJS, 229, 30

McNamara D., 1997, PASP, 109, 1221

McNamara D. H., 2000a, PASP, 112, 1096

McNamara D. H., 2000b, in Breger M., Montgomery M., eds, Astronomical Society of the Pacific Conference Series Vol. 210, Delta Scuti and Related Stars. p. 373

McNamara D. H., 2011, AJ, 142, 110

Moskalik P., 1985, Acta Astronomica, 35, 229

Murphy S. J., 2014, PhD thesis, Jeremiah Horrocks Institute, University of Central Lancashire, UK

Murphy S. J., Shibahashi H., Kurtz D. W., 2013a, MNRAS, 430, 2986

Murphy S. J., et al., 2013b, MNRAS, 432, 2284

Murphy S. J., Hey D., Van Reeth T., Bedding T. R., 2019, MNRAS, 485, 2380

Murphy S. J., Saio H., Takada-Hidai M., Kurtz D. W., Shibahashi H., Takata M., Hey D. R., 2020, MNRAS, 498, 4272

Nemec J. M., Balona L. A., Murphy S. J., Kinemuchi K., Jeon Y.-B., 2017, MNRAS, 466, 1290

Niemczura E., et al., 2015, MNRAS, 450, 2764

Pamyatnykh A. A., 1999, Acta Astron., 49, 119

Pamyatnykh A. A., 2000, in Breger M., Montgomery M., eds, Astronomical Society of the Pacific Conference Series Vol. 210, Delta Scuti and Related Stars. p. 215 (arXiv: astro-ph/0005276)

Pamyatnykh A. A., Dziembowski W. A., Handler G., Pikall H., 1998, A\&A, 333,141

Pápics P. I., 2012, Astronomische Nachrichten, 333, 1053

Pápics P. I., et al., 2012, A\&A, 542, A55

Percy J. R., Matthews J. M., Wade J. D., 1980, A\&A, 82, 172

Petersen J. O., 1973, A\&A, 27, 89

Petersen J. O., Christensen-Dalsgaard J., 1996, A\&A, 312, 463

Pigulski A., Pojmański G., Pilecki B., Szczygieł D. M., 2009, Acta Astron., 59,33

Poleski R., et al., 2010, Acta Astron., 60, 1

Pollacco D. L., et al., 2006, PASP, 118, 1407

Poretti E., 2003, A\&A, 409, 1031

Ricker G. R., et al., 2015, Journal of Astronomical Telescopes, Instruments, and Systems, 1, 014003

Rizzuto A. C., Vanderburg A., Mann A. W., Kraus A. L., Dressing C. D., Agüeros M. A., Douglas S. T., Krolikowski D. M., 2018, AJ, 156, 195

Rodríguez E., Breger M., 2001, A\&A, 366, 178

Rodríguez E., López de Coca P., Costa V., Martín S., 1995, A\&A, 299, 108

Rodríguez E., López-González M. J., López de Coca P., 2000, A\&AS, 144, 469

Rodríguez E., et al., 2007, A\&A, 471, 255

Shulyak D., Tsymbal V., Ryabchikova T., Stütz C., Weiss W. W., 2004, A\&A, 428, 993

Smalley B., 1993, A\&A, 274, 391

Smalley B., 2005, Memorie della Societa Astronomica Italiana Supplementi, 8,130

Smith J. C., et al., 2012, PASP, 124, 1000

Soszyński I., et al., 2008, Acta Astron., 58, 163

Stellingwerf R. F., 1978, AJ, 83, 1184

Stellingwerf R. F., 1979, ApJ, 227, 935

Sterken C., 2005, in Sterken C., ed., Astronomical Society of the Pacific Conference Series Vol. 335, The Light-Time Effect in Astrophysics: Causes and cures of the O-C diagram. p. 3

Stumpe M. C., et al., 2012, PASP, 124, 985

Tamuz O., Mazeh T., Zucker S., 2005, MNRAS, 356, 1466

Thompson K., Coates D. W., 1991, Proceedings of the Astronomical Society of Australia, 9, 281

Tkachenko A., 2015, A\&A, 581, A129

Tkachenko A., Lehmann H., Smalley B., Uytterhoeven K., 2013, MNRAS, 431,3685

Tsymbal V., 1996, in Adelman S. J., Kupka F., Weiss W. W., eds, Astronomical Society of the Pacific Conference Series Vol. 108, M.A.S.S., Model 
Table A1. Additional independent frequencies extracted from the 4-yr LC Kepler data of KIC 5950759 (i.e. after $\nu_{1}$ and $\nu_{2}$ and all their significant harmonics and combinations have been removed). Pulsation phases were calculated with respect to the time zero-point $t_{0}=2455688.770$ BJD (i.e. the midpoint of the 4-yr LC light curve).

\begin{tabular}{ccr}
\hline $\begin{array}{c}\text { Frequency } \\
\left(\mathrm{d}^{-1}\right)\end{array}$ & $\begin{array}{c}\text { Amplitude } \\
(\mathrm{mmag})\end{array}$ & \multicolumn{1}{c}{$\begin{array}{c}\text { Phase } \\
(\mathrm{rad})\end{array}$} \\
\hline $0.31925 \pm 0.00004$ & $0.284 \pm 0.030$ & $1.99 \pm 0.11$ \\
$16.00000 \pm 0.00006$ & $0.202 \pm 0.030$ & $2.03 \pm 0.15$ \\
$18.45070 \pm 0.00009$ & $0.125 \pm 0.030$ & $-0.17 \pm 0.24$ \\
$18.96828 \pm 0.00008$ & $0.149 \pm 0.030$ & $1.47 \pm 0.20$ \\
$20.34427 \pm 0.00005$ & $0.229 \pm 0.030$ & $2.24 \pm 0.13$ \\
$21.15701 \pm 0.00005$ & $0.211 \pm 0.030$ & $2.25 \pm 0.14$ \\
$21.49656 \pm 0.00004$ & $0.291 \pm 0.030$ & $1.69 \pm 0.10$ \\
$22.62858 \pm 0.00003$ & $0.442 \pm 0.030$ & $-2.44 \pm 0.07$ \\
$23.70270 \pm 0.00006$ & $0.187 \pm 0.030$ & $-0.97 \pm 0.16$ \\
$23.82188 \pm 0.00006$ & $0.192 \pm 0.030$ & $0.06 \pm 0.16$ \\
$24.67230 \pm 0.00003$ & $0.380 \pm 0.030$ & $-1.32 \pm 0.08$ \\
$25.36702 \pm 0.00004$ & $0.295 \pm 0.030$ & $-1.57 \pm 0.10$ \\
\hline
\end{tabular}

Table A2. Additional independent frequencies extracted from the residual 31-d SC Kepler data of KIC 5950759 (i.e. after $\nu_{1}$ and $\nu_{2}$ and all their significant harmonics and combinations have been removed). Pulsation phases were calculated with respect to the time zero-point $t_{0}=2455200.895 \mathrm{BJD}$ (i.e. the midpoint of the 31-d SC light curve).

\begin{tabular}{ccr}
\hline $\begin{array}{c}\text { Frequency } \\
\left(\mathrm{d}^{-1}\right)\end{array}$ & $\begin{array}{c}\text { Amplitude } \\
(\mathrm{mmag})\end{array}$ & \multicolumn{1}{c}{$\begin{array}{c}\text { Phase } \\
(\mathrm{rad})\end{array}$} \\
\hline $15.9977 \pm 0.0019$ & $0.42 \pm 0.05$ & $1.46 \pm 0.11$ \\
$18.4513 \pm 0.0018$ & $0.47 \pm 0.05$ & $1.63 \pm 0.10$ \\
$18.9699 \pm 0.0018$ & $0.45 \pm 0.05$ & $0.91 \pm 0.10$ \\
$20.3421 \pm 0.0014$ & $0.58 \pm 0.05$ & $-0.62 \pm 0.08$ \\
$21.1629 \pm 0.0017$ & $0.48 \pm 0.05$ & $1.95 \pm 0.10$ \\
$21.4957 \pm 0.0007$ & $1.19 \pm 0.05$ & $-2.47 \pm 0.04$ \\
$22.6277 \pm 0.0012$ & $0.69 \pm 0.05$ & $-2.24 \pm 0.07$ \\
$23.7050 \pm 0.0021$ & $0.39 \pm 0.05$ & $-1.18 \pm 0.12$ \\
$23.8250 \pm 0.0012$ & $0.71 \pm 0.05$ & $-0.84 \pm 0.06$ \\
$24.6676 \pm 0.0009$ & $0.88 \pm 0.05$ & $-1.03 \pm 0.05$ \\
$25.3680 \pm 0.0016$ & $0.51 \pm 0.05$ & $-0.75 \pm 0.09$ \\
$28.3737 \pm 0.0015$ & $0.56 \pm 0.05$ & $0.57 \pm 0.08$ \\
$33.6469 \pm 0.0013$ & $0.64 \pm 0.05$ & $2.83 \pm 0.07$ \\
\hline
\end{tabular}

Atmospheres and Spectrum Synthesis. p. 198

Uytterhoeven K., et al., 2011, A\&A, 534, A125

Van Reeth T., et al., 2015, A\&A, 574, A17

Virtanen P., et al., 2020, Nature Methods, 17, 261

Walraven T., Walraven J., Balona L. A., 1992, MNRAS, 254, 59

Watson R. D., 1988, Ap\&SS, 140, 255

Yang X. H., Fu J. N., Zha Q., 2012, AJ, 144, 92

Yang T., et al., 2018, ApJ, 863, 195

Ziaali E., Bedding T. R., Murphy S. J., Van Reeth T., Hey D. R., 2019, MNRAS, 486, 4348

Zorec J., Royer F., 2012, A\&A, 537, A120

van der Walt S., Colbert S. C., Varoquaux G., 2011, Computing in Science Engineering, 13, 22

\section{APPENDIX A: FREQUENCY LISTS}

The additional independent non-radial pulsation mode frequencies identified in the LC and SC Kepler data of KIC 5950759 are provided in Tables A1 and A2, respectively.
This paper has been typeset from a $\mathrm{T}_{\mathrm{E}} \mathrm{X} / \mathrm{L} \mathrm{T}_{\mathrm{E}} \mathrm{X}$ file prepared by the author. 